\title{
Development
}

\section{Notch Signaling between Cerebellar Granule Cell Progenitors}

\author{
DToma Adachi, ${ }^{1,2}$ - Satoshi Miyashita, ${ }^{1}$ Mariko Yamashita, ${ }^{1,3}$ Mana Shimoda, ${ }^{1,2}$ \\ (1)Konstantin Okonechnikov, ${ }^{4,5}$ Lukas Chavez, ${ }^{6,7}$ Marcel Kool, ${ }^{4,5,8}$ Stefan M. Pfister, ${ }^{4,5,9}$ \\ (1)Takafumi Inoue, ${ }^{2}$ DDaisuke Kawauchi, ${ }^{1}$ and Mikio Hoshino'
}

\section{https://doi.org/10.1523/ENEURO.0468-20.2021}

\begin{abstract}
${ }^{1}$ Department of Biochemistry and Cellular Biology, National Institute of Neuroscience, National Center of Neurology and Psychiatry, Tokyo 187-8551, Japan, ${ }^{2}$ Department of Life Science and Medical Bioscience, School of Advanced Science and Engineering, Waseda University, Tokyo 169-8555, Japan, ${ }^{3}$ Department of NCNP Brain Function and Pathology, Graduate School of Medical and Dental Sciences, Tokyo Medical and Dental University, TMDU, Tokyo 113-8510, Japan, ${ }^{4}$ Hopp-Children's Cancer Center Heidelberg (KiTZ), 69120 Heidelberg, Germany, ${ }^{5}$ Division of Pediatric Neurooncology, German Cancer Research Center (DKFZ) and German Cancer Consortium (DKTK), 69120 Heidelberg, Germany, ${ }^{6}$ Department of Medicine, University of California San Diego, La Jolla, CA 92093, ${ }^{7}$ Moores Cancer Center, University of California San Diego, La Jolla, CA 92093, ${ }^{8}$ Princess Maxima Center for Pediatric Oncology, 3584 CS Utrecht, The Netherlands, and ${ }^{9}$ Department of Pediatric Hematology and Oncology, Heidelberg University Hospital, 69120 Heidelberg, Germany
\end{abstract}

\section{Visual Abstract}

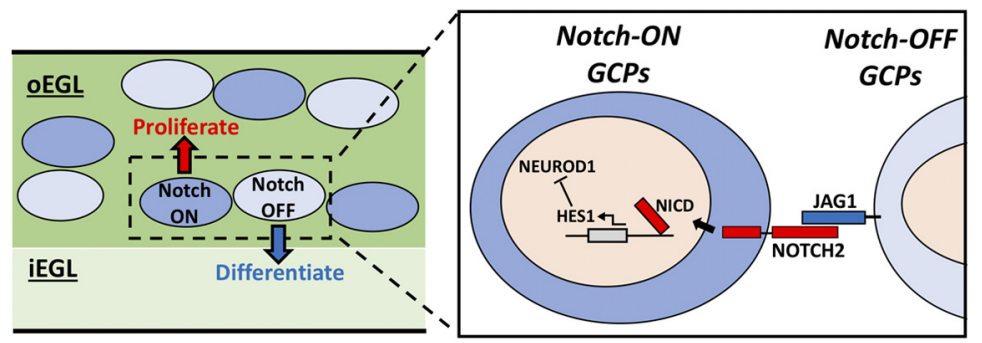

Cerebellar granule cells (GCs) are cells which comprise over $50 \%$ of the neurons in the entire nervous system. GCs enable the cerebellum to properly regulate motor coordination, learning, and consolidation, in addition to cognition, emotion and language. During GC development, maternal GC progenitors (GCPs) divide to produce not only postmitotic GCs but also sister GCPs. However, the molecular machinery for regulating the proportional production of distinct sister cell types from seemingly uniform GCPs is not yet fully understood. Here we report that Notch signaling creates a distinction between GCPs and leads to their proportional

\section{Significance Statement}

This study is the first to succeed in visualization of Notch signaling in vivo during cerebellar development. Granule cell progenitors (GCPs) in the outermost layer of the developing cerebellum are a seemingly homogenous cell population, but this study revealed two types of GCPs; more proliferative Notch-ON-GCPs and more differentiative Notch-OFF-GCPs, the latter of which gradually give rise to postmitotic GCs. Our experiments suggest that NOTCH2 and HES1 are involved cell-autonomously to suppress GCP differentiation by inhibiting NEUROD1 expression. In contrast, JAG1-expressing cells non-autonomously upregulated Notch signaling activities via NOTCH2-HES1 in surrounding GCPs, suppressing their differentiation. This study gives new insights into the mechanisms controlling the differences within homogenous cell populations that direct proper and coordinated cell differentiation. 
differentiation in mice. Among Notch-related molecules, Notch1, Notch2, Jag1, and Hes1 are prominently expressed in GCPs. In vivo monitoring of Hes1-promoter activities showed the presence of two types of GCPs, Notch-signaling ON and OFF, in the external granule layer (EGL). Single-cell RNA sequencing (scRNA-seq) and in silico analyses indicate that ON-GCPs have more proliferative and immature properties, while OFFGCPs have opposite characteristics. Overexpression as well as knock-down (KD) experiments using in vivo electroporation showed that NOTCH2 and HES1 are involved cell-autonomously to suppress GCP differentiation by inhibiting NEUROD1 expression. In contrast, JAG1-expressing cells non-autonomously upregulated Notch signaling activities via NOTCH2-HES1 in surrounding GCPs, eventually suppressing their differentiation. These findings suggest that Notch signaling results in the proportional generation of two types of cells, immature and differentiating GCPs, which contributes to the well-organized differentiation of GCs.

Key words: cerebellar granule cell progenitor; Notch signaling

\section{Introduction}

Notch signaling is one of the most important signaling pathways involved in many aspects of life (Tsakonas et al., 1999; Andersson et al., 2011). It is mainly known for the intercellular signaling between "signal-sending cells," which present the Notch ligand on their cell surfaces and "signal-receiving cells," which present the Notch receptor (Tsakonas et al., 1999; Andersson et al., 2011). Once the ligand binds to the receptor, the downstream intracellular pathway is activated in the Notch receptor-presenting cells, upregulating expression of Hes and Hey family transcription factors, and subsequently affecting the binary cell fate of adjacent cells (Tsakonas et al., 1999; Andersson et al., 2011).

Notch signaling molecules were first identified as neurogenic genes in Drosophila via systemic genetic screening by the Campos-Ortega group (Lehmann et al., 1983). They identified Notch, $\delta$, master mind, Enhancer of Split, which later turned out to constitute a very important signaling pathway, the "Notch signaling pathway." In Drosophila embryogenesis, neuroectodermal cells stochastically differentiate into epidermal cells and neuroblasts in the ratio of 4:1 (Campos-Ortega and Hartenstein, 1985). However, if a neurogenic gene is disrupted, all neuroectodermal cells differentiate into neuroblasts (Lehmann et al., 1983; Tsakonas et al., 1999). This observation led to the notion that neurogenic genes (or Notch signaling) are

\footnotetext{
Received November 2, 2020; accepted March 9, 2021; First published March 24, 2021.

The authors declare no competing financial interests.

Author contributions: T.A., S.M., L.C., M.K., S.M.P., T.I., D.K., and M.H. designed research; T.A., S.M., M.Y., and M.S. performed research; T.A., S.M., K.O., and D.K. analyzed data; T.A. and M.H. wrote the paper.

This work was supported by Japan Society for the Promotion of Science Grants $18 \mathrm{H} 02538$ and $16 \mathrm{H} 06528$ (to M.H.), $20 \mathrm{~K} 15919$ (to S.M.), and 18J20309 (to T.A.); the Japan Agency for Medical Research and Development Grant JP20dm0107085h0001 (to M.H.); Intramural Research Grants 27-7 and 28-4 (to M.H.); and the Heidelberg Center for Personalized Medicine Grant H065.

Acknowledgements: We thank Dr. R. Kageyama, Dr. I. Imayoshi, and Dr. M. Yamada (Kyoto University) for pHes1-d2GFP, pHes5-d2GFP, and Hes1pvenus vectors and $R$. Yu for valuable comments.

Correspondence should be addressed to Satoshi Miyashita at miyasatoshi@ ncnp.go.jp or Mikio Hoshino at hoshino@ncnp.go.jp.

https://doi.org/10.1523/ENEURO.0468-20.202 Copyright $\odot 2021$ Adachi et al.
}

This is an open-access article distributed under the terms of the Creative Commons Attribution 4.0 International license, which permits unrestricted use, distribution and reproduction in any medium provided that the original work is properly attributed. involved in "lateral inhibition," by which only a limited number of cells can differentiate into a neural lineage whereby the rest go to an epidermal lineage. Thus, the Notch signaling machinery or lateral inhibition system enables uniform cells to differentiate proportionally into different cell types.

Since then, numerous studies on Notch signaling have identified identical or similar genes in vertebrates, including Notch1-4, DII1,2,4, Jag1,2, Maml1,2,3, Hes1-7, and Hey1,2 (Kageyama et al., 2007; Andersson et al., 2011). Notch signaling is involved in various developmental events in various tissues, including the nervous system not only in invertebrates but also in vertebrates (Louvi and Tsakonas, 2006). For example, Notch signaling is involved in the production of neurons and neural progenitors from radial glia of the mammalian cerebral cortex, precisely regulating the ratio of sister radial glia and sister neuronal cells (Shitamukai et al., 2011). Thus, it is believed that one of the important functions of Notch signaling is to generate different types of cells from uniform cells, which may be the universal basis for the development of multicellular organisms that originated from a single cell, that is, an oocyte or an egg.

The cerebellum contains a tremendous number of granule cells (GCs) that comprise over $50 \%$ of the neurons in the entire nervous system (Williams and Herrup, 1988). Numerous GCs enable the cerebellum to properly regulate motor coordination, motor learning, and consolidation, in addition to cognition, emotion and language (Lackey et al., 2018). GC progenitors (GCPs) are mitotic cells located in the outer external GC layer (oEGL) of the developing cerebellum (Chizhikov and Millen, 2003). Although GCPs in the oEGL seem to be uniform, they divide to produce two types of sister cells, GCPs and GCs (Yang et al., 2015). GCs generated from GCPs move to the inner EGL (iEGL) and then migrate radially through the molecular layer (ML) to reach the inner GC layer (IGL; Schilling, 2018). The proportion of sister GCPs and GCs is thought to be precisely regulated at each developmental stage (Miyashita et al., 2017). Disruption of this regulation may cause a smaller cerebellum as observed in cerebellar hypoplasia (Basson and Wingate, 2013). It may also lead to generation of $\mathrm{SHH}$ subgroup medulloblastoma, a tumor derived from GCPs (Goodrich et al., 1997).

Several reports have suggested expression of some Notch related molecules in GCPs/GCs (Tanaka et al., 1999; Irvin et al., 2001, 2004; Solecki et al., 2001; Stump et al., 2002; Tanaka and Marunouchi, 2003; Eiraku et al., 
2005). The Solecki group showed that overexpression of $\mathrm{NOTCH} 2$ and HES1 suppressed the differentiation of GCs as was estimated by neurite length in cultured GCPs/GCs as well as cerebellar explant (Solecki et al., 2001). However, there have been no studies that directly observed Notch signaling between GCPs or that showed the significance of Notch signaling between GCPs for GC development.

In this study, we identified Notch signaling molecules that are abundantly expressed in GCPs. Furthermore, we succeeded in classifying GCPs into Notch-ON and -OFF cells, the former of which are more immature and proliferative, whereas the latter are less proliferative and more differentiated. Intercellular interaction of JAG1 and NOTCH2 increase HES1 expression in NOTCH2-expressing cells, which eventually drive the cells to be Notch-ON cells. NOTCH2 and HES1 are autonomously involved in keeping GCPs in a proliferative state, while JAG1-expressing cells had similar effects non-autonomously. We also found that HES1 suppresses the expression of NEUROD1, an important factor that promotes GC differentiation, suggesting that Notch-signaling causes GCPs to remain in a proliferative state by suppressing NEUROD1 expression. This study reveals a new function of Notch signaling in GC development and provides insights into the machinery underlying how different cell types are proportionally generated from uniform cells.

\section{Materials and Methods}

\section{Animals}

All mouse experiments were approved by the Animal Care and Use Committee of the National Institute of Neuroscience, Japan. Mice were housed in SPF conditions and maintained on a 12/12 h light/dark cycle with free access to food and water. ICR pups were obtained from SLC. In all analyses, we used pups without identifying the sex of each animal.

\section{Quantitative PCR (qPCR)}

GCP isolation and qPCR was performed as previously described (Kutscher et al., 2020). In brief, 5 million GCPs were plated per well in a six-well plate and cultured for 48 $\mathrm{h}$ with or without $200 \mathrm{~mm}$ smoothened agonist (SAG, Merck). GCPs were harvested and cDNA was generated with Superscript Ilkit (Invitrogen). Relative gene expression was compared with the geometric mean of Hrpt1, Rp/27, and Rer1 (Thomas et al., 2014). The primer sequences used are as following:

Hrpt1: CAAACTTTGCTTTCCCTGGT and TCTGGCC TGTATCCAACACTTC,

Rp/27: AAGCCGTCATCGTGAAGAACA and CTTGAT CTTGGATCGCTTGGC,

Rer1: GCCTTGGGAATTTACCACCT and CTTCGAA TGAAGGGACGAAA,

Cond2: GAGAAGCTGCCCTGATCCGCA and CTTC CAGTTGCAATCATCATCGACG,

Notch1: GCTGCCTCTTTGATGGCTTCGA and CACA TTCGGCACTGTTACAGCC,
Notch2: CCACCTGCAATGACTTCATCGG and TCGAT GCAGGTGCCTCCATTCT,

Notch3: GGTAGTCACTGTGAACACGAGG and CAACT GTCACCAGCATAGCCAG,

Notch4: GGAGATGTGGATGAGTGTCTGG and TGGC TCTGACAGAGGTCCATCT,

Hes 1: GGAAATGACTGTGAAGCACCTCC and GAAG CGGGTCACCTCGTTCATG,

Hes5: CCGTCAGCTACCTGAAACACAG and GGTC AGGAACTGTACCGCCTC,

Jag1: TGCGTGGTCAATGGAGACTCCT and TCGC ACCGATACCAGTTGTCTC,

Jag2: CGCTGCTATGACCTGGTCAATG and TGTA GGCGTCACACTGGAACTC,

DII1: GCTGGAAGTAGATGAGTGTGCTC and CACA GACCTTGCCATAGAAGCC,

DII3: CCAGCACTGGATGCCTITTACC and ACCT CACATCGAAGCCCGTAGA,

DII4: GGGTCCAGTTATGCCTGCGAAT and TTCGG CTTGGACCTCTGTTCAG.

\section{Plasmids}

Expression vectors of HES1, 5, JAG1, and NEUROD1, and sh vectors for Notch1, Notch2, Hes1, Hes5, Jag1, and NeuroD1 were constructed as previously reported (Kawauchi et al., 2006). All cloned expression fragments were inserted into a pCAGGS vector (GE Healthcare). The primers sequences, used for cloning are, for HES1: 5'ATGCCAGCTGATATAATGG-3' and 5'-TCATCCTCTG GTCCGCT-3', HES5: 5'-ATGGCCCCAAGTACCGT-3' and 5'-TCATCCTCTGGTCCGCT-3', JAG1: 5'-TCCAC GGAGTATATTAGAGCC-3', 5'-GCTAGCACACTCATCGA TG-3', 5'-AACCCCTGCTTGAATGGG-3' and 5'-CTATAC GATGTATTCCATCCGGTT-3', and NEUROD1: 5'-ATGA CCAAATCATACAGCGA-3' and 5'-CTAATCGTGAAAGA TGGCAT- $3^{\prime}$. sh vectors were generated by inserting the double-stranded oligonucleotides into a mU6 pro vector. The targeting sequence for each vector was designed by siDirect 2.0 (Naito et al., 2009). Sequences are, Notch1: \#1 5'-AAGGTGTATACTGTGAAATCAAC-3', \#2 5' -CTGTA ACAGTGCCGAATGTGAGT-3', Notch2: \#1 5'- AGGCCT TAATTGTGAAATTAATT-3', \#2 5'-GAGGTGATAGGC TCTAAGATATT-3', Hes1: \#1 5'-GAGGCGAAGGGCAAG AATAAATG-3', \#2 5'-TTGGATGCACTTAAGAAAGATAG3', Hes5: \#1 5'-CCGCATCAACAGCAGCATAGAGC-3', \#2 5'-CCGTCAGCTACCTGAAACACAGC-3', NeuroD1: \#1 5'-GCCTAGAACGTITTAAATTAAGG-3', \#2 5'-TGGC AACTTCTCTTTCAAACACG-3'. pCAG-H2BGFP vectors and pCAG-mCherry were a gift from N. Masuyama. pHes1-d2GFP, pHes5-d2GFP and Hes1p-venus vectors were gifts from R. Kageyama (Kohyama et al., 2005; Ohtsuka et al., 2006). pCAG-H2B-BFP (pTagBFP-H2B) was purchased from evrogen (catalog \#FP176). Coding region of Hes1 or Hes5 was inserted into a pEGFP-N3 vector to generate HES1-fusion-GFP and HES5-fusionGFP vectors.

\section{Antibodies and immunohistochemistry}

Detailed protocols for immunohistochemistry were described previously (Seto et al., 2014). Briefly, neonatal 
mice were fixed with 4\% PFA and embedded with O.C.T compound (Sakura Finetek). Frozen brains were sagittally sectioned into $18-\mu \mathrm{m}$ slices with a cryostat (CM3050 S; Leica). Cryosections were incubated at room temperature with $1 \%$ normal donkey serum containing $0.2 \%$ PBST (blocking solution) for $1 \mathrm{~h}$. After blocking, sections were incubated with primary antibodies diluted with blocking solutions at $4^{\circ}$ for $16 \mathrm{~h}$. The following primary antibodies were used, goat anti-Notch1 (1:500; sc-6015; Santa Cruz Biotechnology), goat anti-Notch2 (1:500; sc-7423; Santa Cruz Biotechnology), rabbit anti-Jagged1 (1:500; ab7771; Abcam), chicken anti-GFP (1:1000; GFP-1010; Aves), rabbit anti-RFP (1:500; PM005; MBL; RFP antibody was used to enhance CAG-mCherry signals), rabbit anti-Atoh1 (1:200; Yamada et al., 2014), rat anti-Ki67 (1:500; 14-569882; eBioscience), rabbit anti-Pax6 (1:500; PRB-278P; BioLegend), and goat anti-NeuroD (N-19; 1:200; sc-1084; Santa Cruz Biotechnology). Subsequently, slides were rinsed with PBS and incubated with secondary antibodies conjugated with Alexa Fluor 488, Alexa Fluor 568, or Alexa Fluor 647 (1:400; Abcam) and DAPI ( $25 \mu \mathrm{g} / \mathrm{ml}$; Invitrogen) in blocking buffer in PBS at room temperature for $2 \mathrm{~h}$. Slides were rinsed with PBS again and mounted with Permafluor.

\section{In vivo electroporation}

in vivo electroporation to neonatal mice was described previously (Owa et al., 2018). Expression plasmids were diluted to $1 \mu \mathrm{g} / \mu \mathrm{l}$, shRNAs to $2 \mu \mathrm{g} / \mu \mathrm{l}$ and fluorescent protein vectors to $0.5 \mu \mathrm{g} / \mu \mathrm{l}$ in milliQ. pHes1-d2GFP, pHes5-d2GFP and Hes1p-venus vectors were diluted to $1 \mu \mathrm{g} / \mu \mathrm{l}$ in milliQ. Fastgreen was added to visualize the plasmid solution. Pups were anesthetized on ice before electroporation; $10-\mu$ l plasmid solutions were injected into P5 or P6 ICR cerebella over the skull. Electric pulses (50 ms in duration, $80 \mathrm{~V}$, seven times) were delivered to mice with 150-ms interval using forceps-type electrodes (NEPA gene). The pups were kept warm at $37^{\circ}$ until recovered and returned to the litter. Pups were fixed a few days after electroporation as indicated. For "double electroporation" experiments (Fig. 9A-C), pups were temporarily returned to the litter after the first electroporation and the procedure was repeated $6 \mathrm{~h}$ later. In Figure $9 D-F$, EdU $(10 \mathrm{mg} / \mathrm{ml})$ diluted in PBS $(50 \mathrm{mg} / \mathrm{kg}, 20 \mu \mathrm{l}$ total volume) was introduced by intraperitoneal injection immediately following electroporation.

\section{Slice culture and time lapse imaging}

Cerebellar slice culture was prepared as previously reported (Owa et al., 2018). Electroporated postnatal day (P)6 or P7 mouse cerebella were sliced into $250-\mu \mathrm{m}$ sagittal sections with a vibratome. Slices were cultured at $37^{\circ}$ for $6 \mathrm{~h}$, and time lapse imaging was taken with a confocal microscope, FV3000 (Olympus). Pictures were taken every $5 \mathrm{~min}$. In Figure $2 D-F, 1 \mu \mathrm{l}$ DMSO or $\gamma$ secretase inhibitor RO4929097 (5 nM, Selleck) were added to the medium just before the imaging.

\section{Image acquisition and quantification}

All images were acquired from midline vermis region of Lobules IV-VI with confocal microscopy, LSM780 (Carl
Zeiss) and FV3000 (Olympus). Acquired images were analyzed with ImageJ (RSB). The analyses to calculate the rate of undifferentiated states of GCPs were done by using the "Cell counter in Plugins" in ImageJ. All of the GFP (or mCherry)-positive cells in EGL, ML, and IGL were included in the analyses. The analyses to quantify the protein level of GFP and mCherry in Figure 3, and NEUROD1 in Figure 10, were done by using "Measures in Analyze" in ImageJ. The NEUROD1 brightness of GFP negative cells located in the next to GFP-positive cells were used as control.

\section{Cell culture and transfection}

Cell culture and transfection were performed as previously reported (Miyashita et al., 2020). Neuro2a (N2a) cells were cultured in DMEM containing 10\% FBS and $100 \mathrm{U} / \mathrm{ml}$ penicillin-streptomycin. Five million cells were plated per well in a six-well plate and $24 \mathrm{~h}$ after the passage, transfection was performed with transfectin reagent (Bio-Rad); $1 \mu \mathrm{g}$ expression vector plasmid DNA and $2 \mu \mathrm{g}$ sh plasmid DNA were introduced in each well.

\section{Western blotting}

$\mathrm{N} 2 \mathrm{a}$ cells were harvested $48 \mathrm{~h}$ after the transfection. Detailed protocols of western blotting were described previously (Shiraishi et al., 2019). Membranes were incubated with primary antibodies at $4^{\circ}$ overnight. The following primary antibodies were used, rabbit anti- $\beta$-actin (1:1000; MBL), rabbit anti-GFP (1:2000; MBL-598; MBL), and rabbit anti-Jagged1 (1:1000; ab7771; Abcam). After membranes were incubated with secondary antibodies at room temperature for $1 \mathrm{~h}$, HRP substrate (Millipore) was added and immuno-signals were detected by LAS4000 (Fujifilm). In quantification, all of the targeted proteins expression levels are normalized by the expression level of $\beta$-actin.

\section{Smart-Seq [single-cell RNA sequencing (scRNA-seq)]}

Cells were prepared from P7 cerebella using Percoll density gradient centrifugation as described previously (Kawauchi et al., 2012) followed by capturing the cells using the C1 Fluidigm systems with 96-well chips. The total RNAs were extracted from single sorted cells and the library was prepared for the subsequent RNAseq.

\section{Data processing for SCRNA-seq data}

The sequencing reads were aligned to $\mathrm{mm} 10$ reference using STAR 2.4.1d (Dobin et al., 2013). Gene expression counts per cell were computed with HTseq-count tool 0.6.1 (Anders et al., 2015). Quality control for the alignments was performed with Qualimap v2.2.1 (Okonechnikov et al., 2016). The computed counts were further processed with Seurat v3 as described (Stuart et al., 2019). All cells belonging to the cluster 0 (Fig. 4D,F) were categorized by Notch2 expression to Notch2-positive-cells (Notch2>0) and Notch2-negativecells $($ Notch2 $=0)$. Differentially expressed genes $(D E G)$ between Notch2-positive-cells and Notch2-negative cells were determined with DESeq2 (Love et al., 2014). Genes with 
A

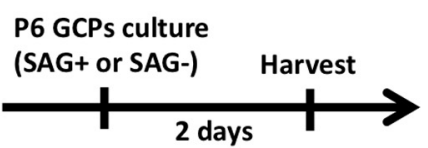

B

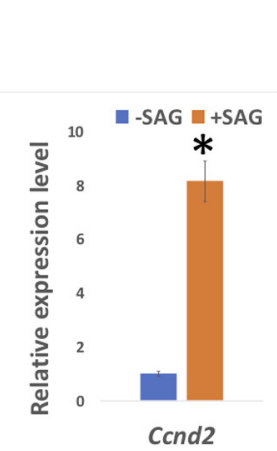

C

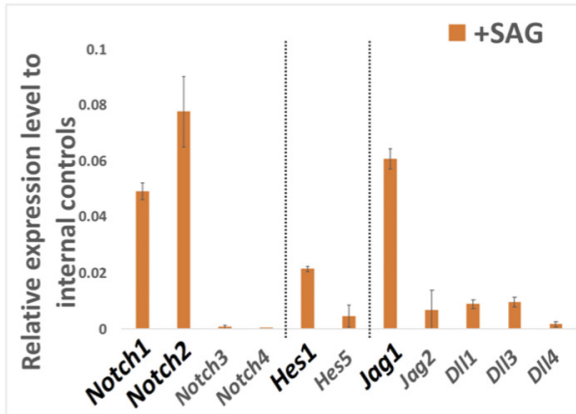

D

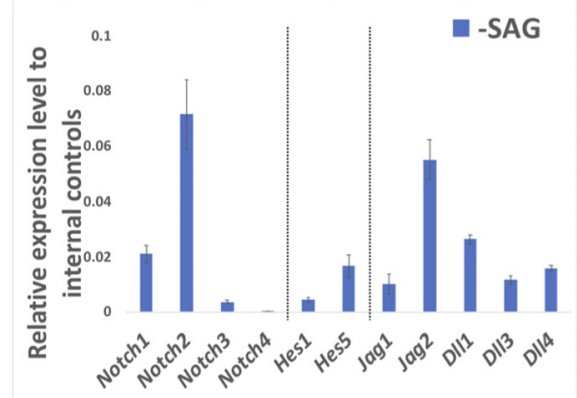

E

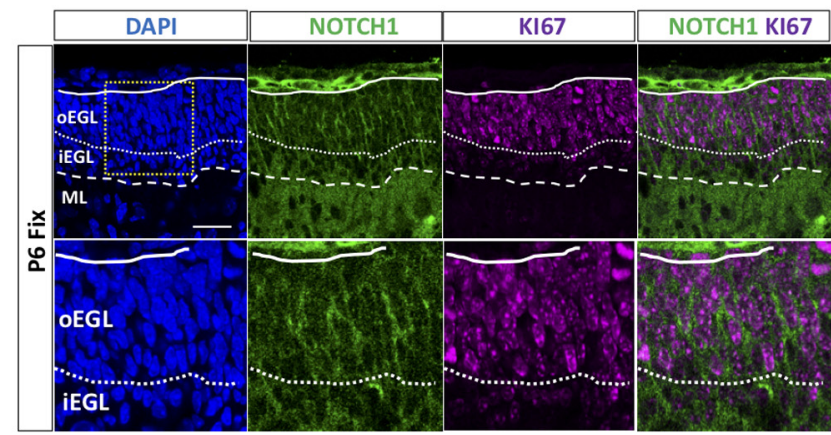

$\mathbf{F}$

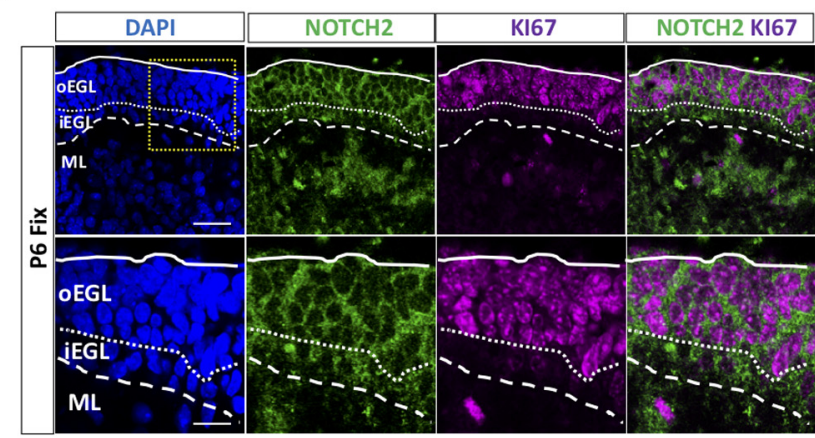

G

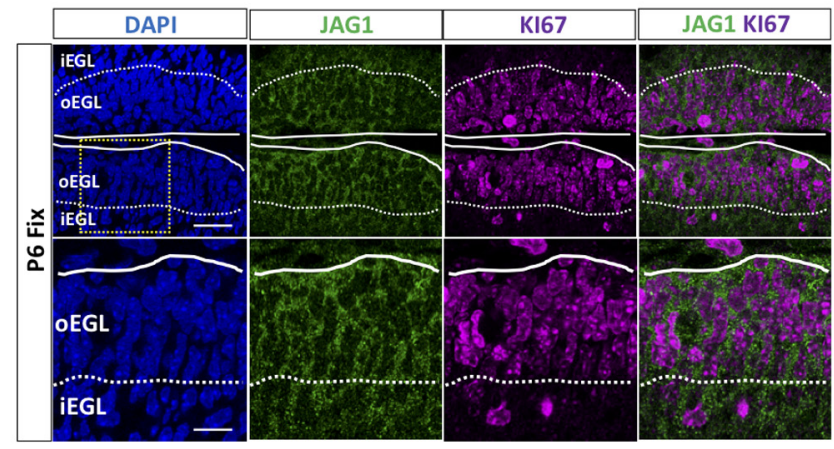

Figure 1. Expression of Notch signaling molecules in cerebellar GCPs. A-D, Gene expression of Notch receptors (Notch1,2,3,4), Hes family genes (Hes1,5), and ligands (Jag1,2, Dll1,3,4) was estimated by quantitative RT-PCR using GCP-like (SAG+) or GC-like (SAG-) cells that were purified from P6 cerebella $(\boldsymbol{A})$. Hrpt1, Rpl27, and Rer1 were used as internal controls $(\boldsymbol{C}, \boldsymbol{D})$. Ccnd2 expression is a marker of GCPs used to monitor the culture conditions $(\boldsymbol{B})$. Data are shown as mean $\pm \mathrm{SEM}$; ${ }^{*} p<0.05$, Student's $t$ test. $\boldsymbol{E}-\boldsymbol{G}$, Immunostaining with indicated antibodies on sagittal cerebellar sections. Sections were co-stained with DAPI, a nuclear marker. Within the EGL, nearly all Ki67-positive cells are GCPs (Chizhikov and Millen, 2003; Yang et al., 2015; Schilling, 2018). Lobule IVN or VI is shown. Differences were not observed as to their expression along an anterior-posterior axis. Scale bars: 30 and $15 \mu \mathrm{m}(\boldsymbol{E}-\mathbf{G})$.

adjusted $p<0.01$ were defined as DEGs (582 upregulated, 74 downregulated).

Unchanged genes were selected from genes with adjusted $p>0.05$. GSEA analysis was performed with the identified DEGs against NOTCH-REACTOME dataset (Molecular Signatures Database v7.1, M10189, Signaling by NOTCH). DAVID was used to conduct the Gene Ontology (GO) analysis (https://david.ncifcrf.gov).

\section{Data availability statement}

scRNA-seq data used in this study were available in Gene Expression Omnibus (GSE153313). 


\section{Code availability}

All the codes used in this study are available from the lead contact (hoshino@ncnp.go.jp).

\section{Statistical analyses}

Individual animals or trials are regarded as biological replicates. All controls were prepared under the same experimental conditions. All data are presented as mean \pm SEM. Statistical tests were performed by Student's $t$ test and one-way ANOVA with Bonferroni's post hoc test; $p$ values were represented as; N.S. for $p>0.05$; ${ }^{*} p<0.05$, ${ }^{\star \star} p<0.01,{ }^{* \star *} p<0.001$.

\section{Results}

\section{Expression of Notch-related molecules in cerebellar GCPs}

Previously, several studies reported that Notch signaling-related molecules were expressed in the cerebellar EGL (Tanaka et al., 1999; Irvin et al., 2001, 2004; Solecki et al., 2001; Stump et al., 2002; Tanaka and Marunouchi, 2003; Eiraku et al., 2005). However, in all cases, no discrimination was made between the expression in GCPs and GCs. Therefore, we isolated GCPs from cerebella at P6 according to our method (Kutscher et al., 2020) and cultured them for $2 \mathrm{~d}$ with or without SAG, an activator of $\mathrm{SHH}$ signaling. Under this condition, SAG-treated and non-treated cells possess characteristics very similar to those of GCPs and GCs, respectively (Kutscher et al., 2020). Consistently, Ccnd2, known as a mitotic GCP marker, were expressed highly in SAG-treated cells, and expressed very low in non-treated cells (Fig. $1 A, B)$. We performed quantitative RT-PCR (qPCR) to determine the expression of Notch signaling-related genes. In SAGtreated cells, or GCP-like cells, Notch1, Notch2, Hes1, and Jag1 were all prominently expressed while expression of Notch3, Notch4, Hes5, Jag2, DII1, DII3, and DII4 were low (Fig. 1C). In SAG-non-treated cells, we observed considerable expression of Notch1, Notch2, Hes5, and Jag2 (Fig. 1D). Because our aim was to investigate the importance of Notch signaling among GCPs in the EGL, we focused on Notch1, Notch2, Hes1, and Jag1 in this study. Next, we performed immunostaining with antibodies against $\mathrm{NOTCH} 1, \mathrm{NOTCH}$, and JAG1 on P6 cerebellum along with KI67, a marker for mitotic cells. NOTCH1, $\mathrm{NOTCH}$, and JAG1, exhibited honeycomb-like staining signals in the oEGL and iEGL (Fig. 1E-G), suggesting that those membrane proteins were expressed by GCPs and GCs in the developing cerebellum.

\section{Notch2-Hes1 signaling is active in the EGL}

Given that some Notch-related molecules were expressed in GCPs in the developing cerebellum, we tried to monitor Notch signaling activities in GCPs in the EGL. To this end, we used vectors, pHes1-d2GFP and pHes5d2GFP, which were designed to express short half-life GFP (d2GFP) under the control of Hes1 and Hes5 promoters, respectively (Ohtsuka et al., 2006). We performed in vivo electroporation (Owa et al., 2018; Chang et al., 2019) with pHes1-d2GFP or pHes5-
d2GFP plus an mCherry-expressing vector (pCAGmCherry) on GCPs in the EGL at P5 and fixed electroporated cerebella $3 \mathrm{~d}$ after electroporation. While pHes1-d2GFP gave rise to significant signals in the EGL, signals for pHes5-d2GFP were barely observed (Fig. $2 A-C$ ). This discrepancy in the promoter activities of Hes 1 and Hes5 in the EGL is consistent with that of the levels of expression strength of Hes 1 and Hes5 in SAG-treated cells (Fig. 1C).

We next tried to confirm whether this Hes1 promoter activity in the EGL is under the control of Notch signaling. For this purpose, we performed time lapse observation of cerebellar slices containing a Hes1 promoter monitoring vector in the presence or absence of RO4929097. RO4929097 is a specific $\gamma$ secretase inhibitor that prevents the cleavage of Notch intracellular domain (NICD), leading to inhibition of its downstream signaling cascades (Luistro et al., 2009). Of note, we used pHes1-Venus instead of pHes1-d2GFP because of requirement of stronger fluorescence signals during time-lapse recordings (Kohyama et al., 2005). We electroporated pHes1-Venus plus pCAG-mCherry to P5 cerebella, generated cerebellar slices at P6 and performed time lapse observations in the presence or absence of RO4929097 for $>8 \mathrm{~h}$ (Fig. 2D-F). The kinetics of the fluorescence intensities clearly showed that RO4929097 gradually but dramatically decreased the Venus intensity reflecting Hes 1 promoter activity, while the Venus intensity was not changed in control (DMSO application) slices during the recordings (Fig. 2D,E). Also, the rate of Venus-positive cells in mCherry + cells were dramatically decreased only in the RO4929097 applied slices (Fig. 2D,F).

These results suggest that fluorescence signals for the Hes 1 promoter in the EGL are regulated by Notch signaling.

Among Notch family genes, Notch1 and Notch2 were strongly expressed in GCP-like cells (Fig. 1C) and in the EGL (Fig. 1E,F). Therefore, we aimed to identify which Notch gene is responsible for the Hes 1 promoter activity in the EGL. For this purpose, we electroporated short hairpin (sh)-RNAs for Notch1 or Notch2 plus pHes1-d2GFP and pCAG-mCherry into P6 cerebella and fixed them at P8 (Fig. 2G). Counting GFP-positive cells in mCherry+ cells showed that knock down (KD) of Notch2 significantly downregulated the Hes1 promoter activity, while KD of Notch1 did not (Fig. 2G,H), suggesting that Notch2-Hes1 signaling is active in the EGL during cerebellar development.

\section{Notch signaling-ON and signaling-OFF GCPs in the oEGL}

We electroporated with pHes1-d2GFP and pCAGmCherry into P5 EGL and immunostained cerebellar samples at P8 with KI67. Interestingly, among electroporated (mCherry-expressing) cells, GFP-positive cells were more mitotic (KI67-positive) than GFP-negative cells (Fig. 3A, $B)$. This suggests that Hes1-promoter active cells tend to remain as proliferating GCPs in the oEGL compared with the negative cells for that activity. In the same experiment, we quantified the fluorescence intensity of GFP in each 
A

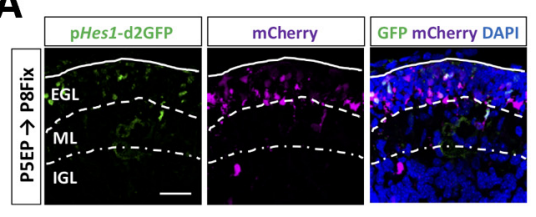

B

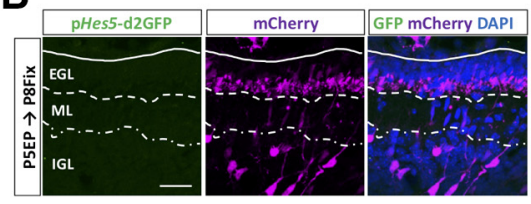

C

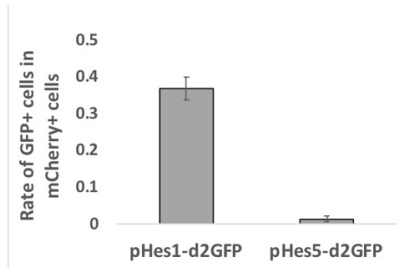

D
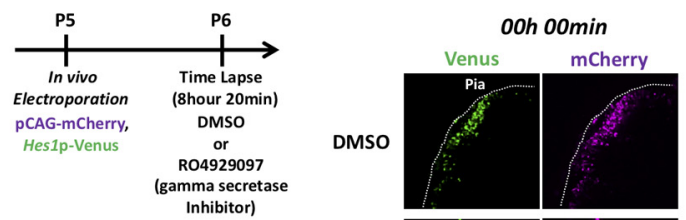

RO4929097
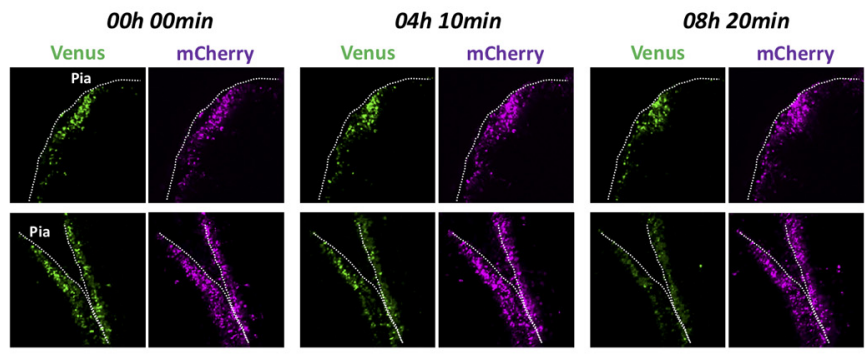

$\mathbf{E}$

$\mathbf{F}$
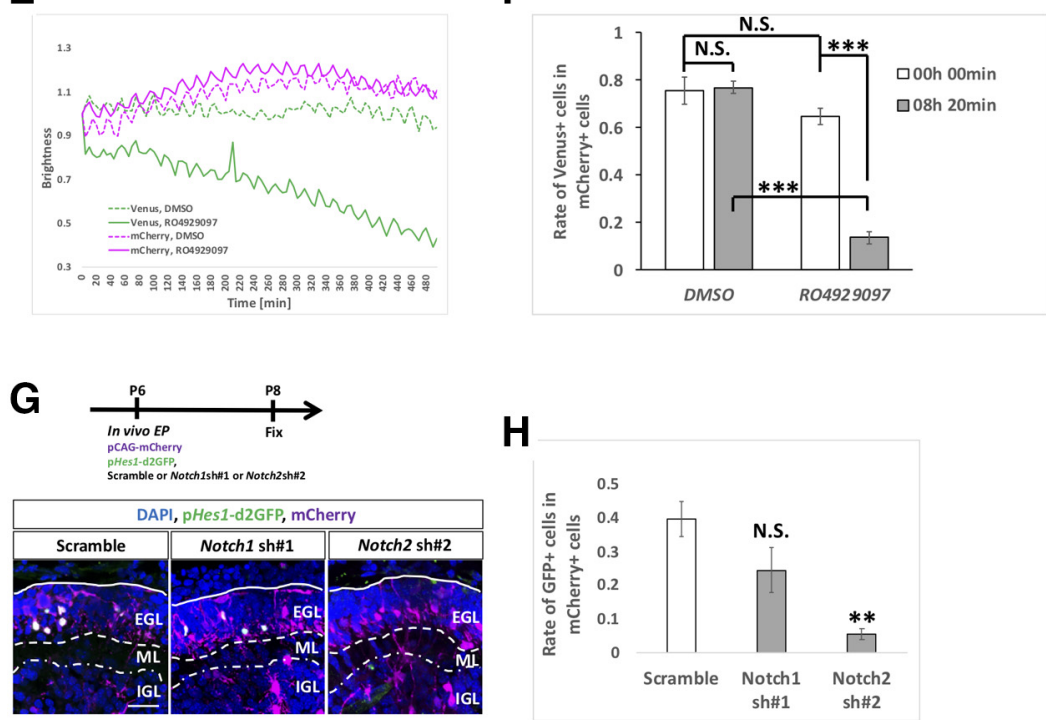

Figure 2. Hes1 promoter activity in EGL is reduced by a $\gamma$ secretase inhibitor and Notch2 KD. A-C, To check the activities of Hes 1 and Hes5 promoters, pHes1-d2GFP $(\boldsymbol{A})$ and pHes5-d2GFP $(\boldsymbol{B})$ were electroporated into P5 EGL with pCAG-mCherry vector. In the EGL, only Hes1 promoter activities were detected $(\boldsymbol{A}-\mathbf{C})$. Rates of GFP-positive cells in mCherry + cells were calculated $(\boldsymbol{C})$. Animal numbers: $N=7$ for pHes1-d2GFP and $N=3$ for pHes5-d2GFP. Scale bars: $30 \mu \mathrm{m}(\boldsymbol{A}, \boldsymbol{B})$. $\boldsymbol{D}-\boldsymbol{F}$, pHes 1 -Venus and pCAG-mCherry were electroporated to P5 mouse, followed by slice culture at P6 $(\boldsymbol{D})$. Time lapse images were taken for $8 \mathrm{~h} 20 \mathrm{~min}$ in the presence of R04929097 ( $\gamma$ secretase inhibitor) or control DMSO that were administered at the start of the time lapse. Decline of the Venus brightness and rate of the Venus-positive cells in mCherry + cells were observed only in the RO4929097 treated slices $(\boldsymbol{D}-\boldsymbol{F})$. Scale bars: $80 \mu \mathrm{m}(\boldsymbol{A})$. Data are shown as mean \pm SEM; ${ }^{* * *} p<0.001$, one-way ANOVA with Bonferroni's post hoc test. Animal numbers: $N=3$ for DMSO and RO4929097. G, $\boldsymbol{H}$, pHes1-d2GFP and pCAG-mCherry were electroporated with indicated KD vectors to P6 cerebella, followed by fixation at P8. KD for Notch2 but not for Notch1 reduced the rates of GFP+ cells in mCherry + cells. Animal numbers: $N=4$ for Notch1 sh\#1 and Notch2 sh\#2. Scale bars: $30 \mu \mathrm{m}(\boldsymbol{F})$. Data are shown as mean \pm SEM; ${ }^{* *} p<0.01$, Student's $t$ test. 
A

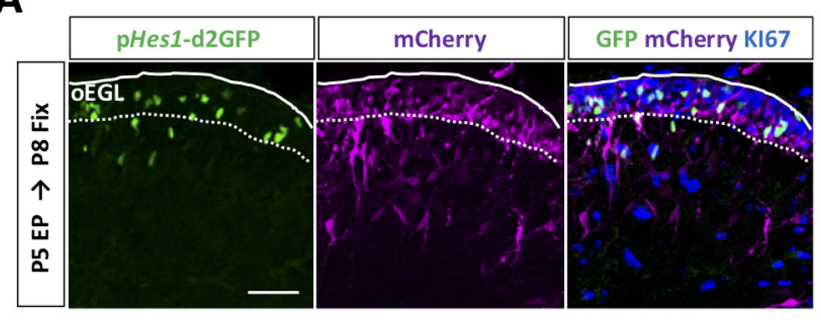

B

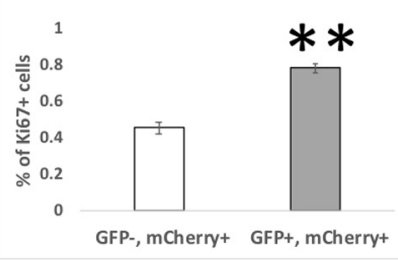

C

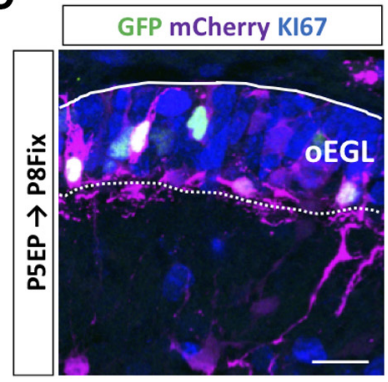

D

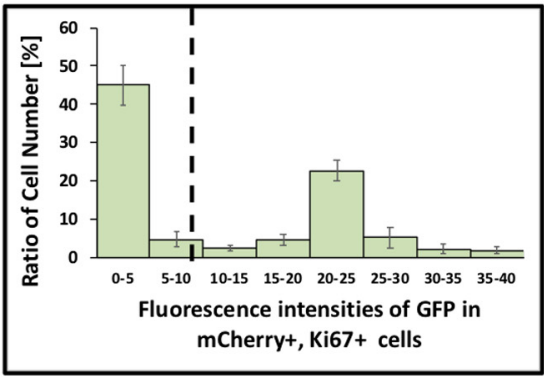

E

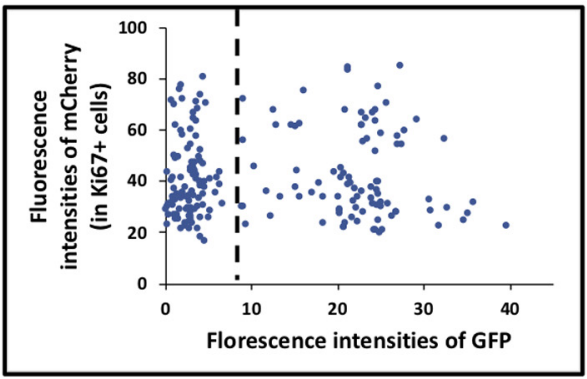

Figure 3. GCPs are divided into two subgroups Notch signaling ON and OFF cells in vivo. A-E, Hes1 promoter activity was stronger in GCPs than GCs $(\boldsymbol{A}, \boldsymbol{B})$. Heterogeneity was observed in Hes1 promoter activities among (KI67-positive) GCPs in the oEGL (C-E). The dashed line represents the threshold of intensity visible by eye $(\boldsymbol{D}, \boldsymbol{E})$. Animal numbers: $N=3$ for the analysis of $\boldsymbol{B}$ and $N=7$ for $\boldsymbol{D}, \boldsymbol{E}$. Scale bars: $45 \mu \mathrm{m}(\boldsymbol{A})$ and $20 \mu \mathrm{m}(\boldsymbol{C})$. Data are shown as mean \pm SEM; ${ }^{* \star} p<0.01$, Student's $t$ test.

GCP (KI67-positive cell) in the oEGL (Fig. 3C-E). Surprisingly, the fluorescence intensities for GFP exhibited a clear bimodal distribution (Fig. 3D,E), while those for control mCherry appeared unimodal (Fig. 3E). This suggests that GCPs in the oEGL comprise two types of cells, ON and OFF cells, for Notch2-Hes1 signaling.

Next, we performed scRNA-seq on primary GCPs collected from P7 cerebella using the Smart-seq technology. From six independent batches, total 109 cells were successfully subjected to RNA-seq. We performed dimensional compression of the obtained gene expression data into a two-dimensional matrix employing the Seurat software and identified three clusters of single cells (Fig. 4A). From the result, it was revealed that GCP and GC markers were highly expressed in the cluster 0 and $1 / 2$ cells, respectively (Fig. 4B,C). As we found that Notch signaling in GCPs is dependent on Notch2 (Fig. 2G,H), we investigated Notch2 expression in the single cells. In the GCP cluster (cluster 0), 29 cells were Notch2-positive, while 15 were negative (Fig. 4D). Although the expression of Jag1 and Hes 1 was detected by quantitative RT-PCR in GCPs (Fig. 1B), their expression was found only in limited number of cells in this scRNA-seq analyses, probably because of the limitation for detecting their transcripts under this experimental condition (Fig. 4E). We further classified the GCPs cluster into two groups, Notch2-positive and Notch2-negative cells, which showed dramatically distinct expression profiles (Fig. 4F).

In the differential expression analysis using the DESeq2 software (Love et al., 2014), we identified 656 DEG between Notch2-positive and Notch2-negative GCPs (Fig. $4 F)$. Expression of 582 genes was upregulated in Notch2positive GCPs, while that of 74 genes was downregulated (Fig. 4F). This clearly suggests that these two populations,
Notch2-positive and Notch2-negative GCPs have quite different molecular characteristics. We performed gene set enrichment analysis (GSEA) to test for a potential enrichment of Notch signaling in Notch2-positive and Notch2-negative GCPs by comparing 656 DEG to the "REACTOME_SIGNALING_BY_NOTCH" gene set in the Molecular Signatures Database v7.1 (M10189, Signaling by $\mathrm{NOTCH}$ ). As a result, we found that highly expressed genes in Notch2-positive/negative GCPs in our data match to the upregulated/downregulated genes in Notch signaling ON cells, significantly (Fig. 4G). We then performed GO analysis for these DEGs (Fig. 4H,I). Several of the significant $\mathrm{GO}$ categories for upregulated genes in Notch2-positive GCPs were related to cell cycle progression (Fig. 4H), while some GOs for upregulated genes in Notch2 negative GCPs implicated cell differentiation events, including cell migration (Fig. 4/). These findings suggest that Notch-signaling ON GCPs are a more proliferative and less differentiated population, while Notch signaling-OFF GCPs are less proliferative and more differentiated.

\section{Notch2-Hes1-dependent Notch signaling maintains GCPs in immature and proliferative state}

We and others previously developed an in vivo electroporation gene transfer method for GCPs during cerebellar development (Owa et al., 2018; Chang et al., 2019). In this study, we further characterized the dynamics of differentiation and migration processes of electroporated cells (Fig. 5). The EGL was electroporated with a nuclear-localizing GFP (pCAG-H2B-GFP) at P5 and fixed at P6, P7, P8, P9, and P12 (Fig. 5A,B). By this method, GCPs facing the cerebellar surface, or the pia mater, were transfected with 
A

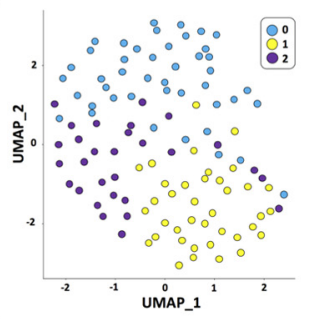

C

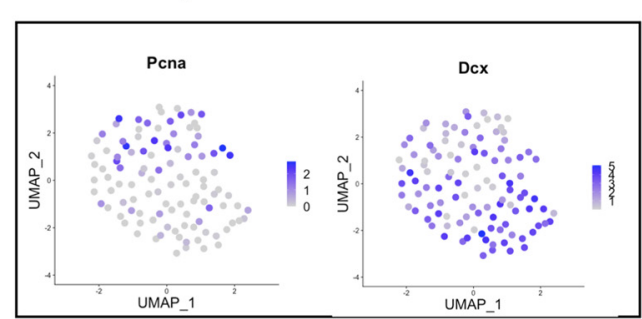

E

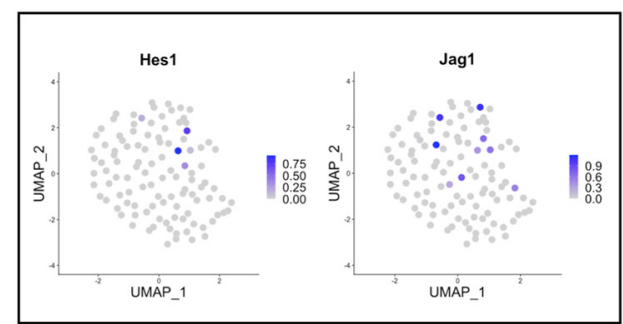

B

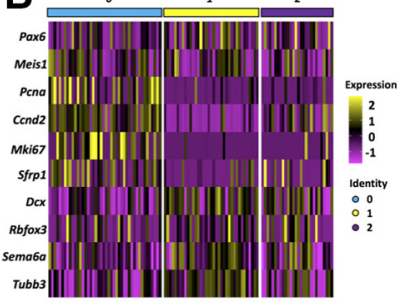

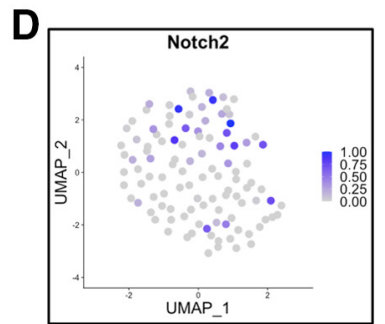

UMÁP_1
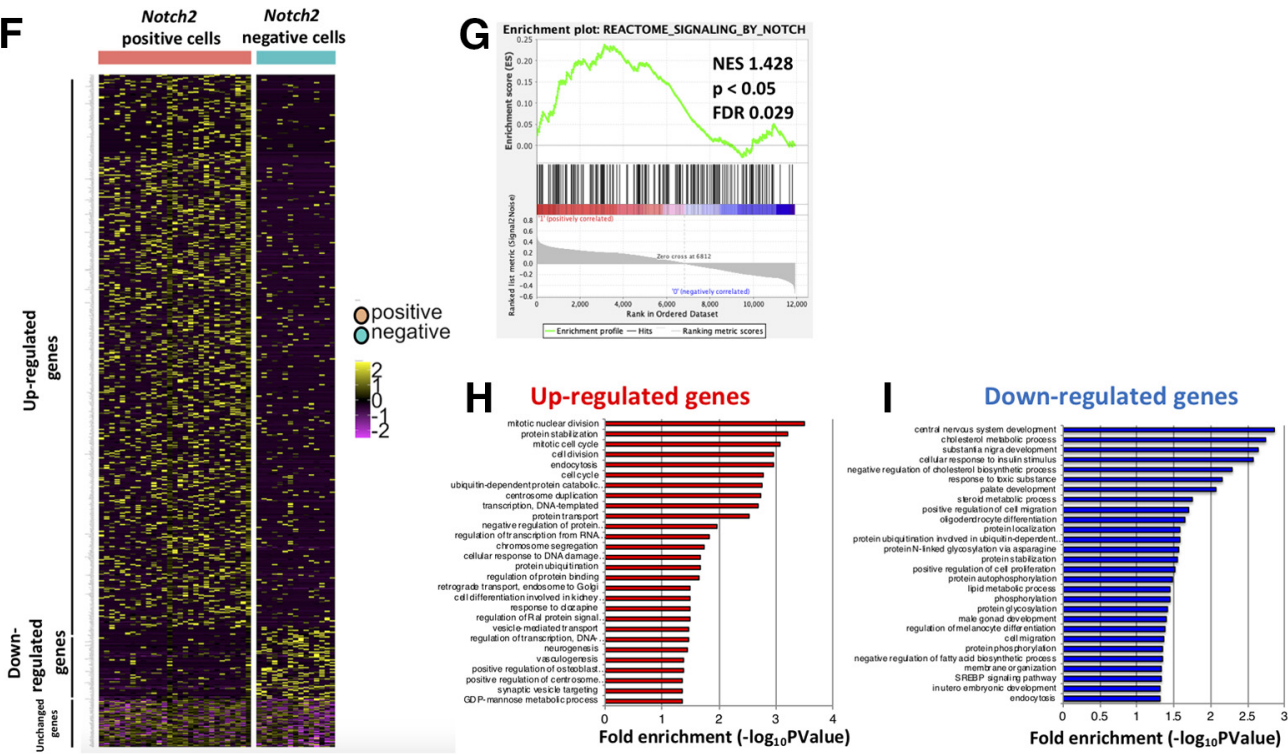

Figure 4. GCPs are divided into two subgroups Notch signaling ON and OFF cells in silico. A, scRNA-seq (Smart-seq) analysis of 109 GCPs purified from P7 mice. Uniform manifold approximation and projection (UMAP) dimensional reduction were performed and three clusters were identified $(0,1,2)$. B. Heatmap for expression of GC-lineage markers (Pax6, Meis 1$)$, GCP markers $(P c n a$, Ccnd2, Mki67, Sfrp1), and GC markers (Dcx, Rbfox3, Sema6a, Tubb3) in cells of distinct clusters. Molecular features of distinct clusters suggested that cluster 0 corresponded to GCPs and cluster 1 and 2 to GCs. $\boldsymbol{C}-\boldsymbol{E}$, Normalized expression of selected genes are visualized onto the UMAP-dimension (C). Pcna (a marker for GCPs) are mostly expressed in Cluster 0, while Dcx (a marker for GCs) is expressed in Cluster1,2. Notch2, Hes1, and Jag1 expressions are also shown (D, E). $\boldsymbol{F}$, GCPs, which were extracted from scRNA-seq of P7 mice (Cluster 0 cells), were grouped by Notch2 expression (positive and negative cells, please also see Materials and Methods). Expression profiles of 656 DEG between Notch2-positive and Notch2-negative GCPs were visualized by the heatmap. Expression of 582 genes were upregulated in Notch2-positive GCPs, while that of 74 genes were downregulated. G, GSEA of upregulated genes versus downregulated genes in Notch2-positive cells was performed with the REACTOME_SIGNALING_BY_NOTCH dataset. NES, normalized enrichment score; FDR, false discovery rate. $\boldsymbol{H}, \boldsymbol{I}$, GO analysis was performed for the 582 upregulated genes in Notch2-positive GCPs $(\boldsymbol{H})$ and for 74 downregulated genes $(\boldsymbol{I})$. 
A

Alectroporate H2B -GFP in P5 Fix in P6,7,8,9,12
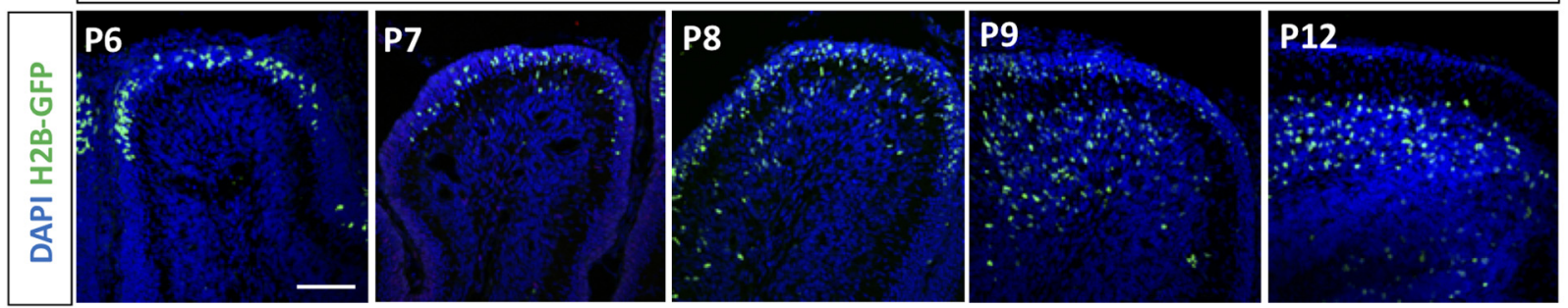

B

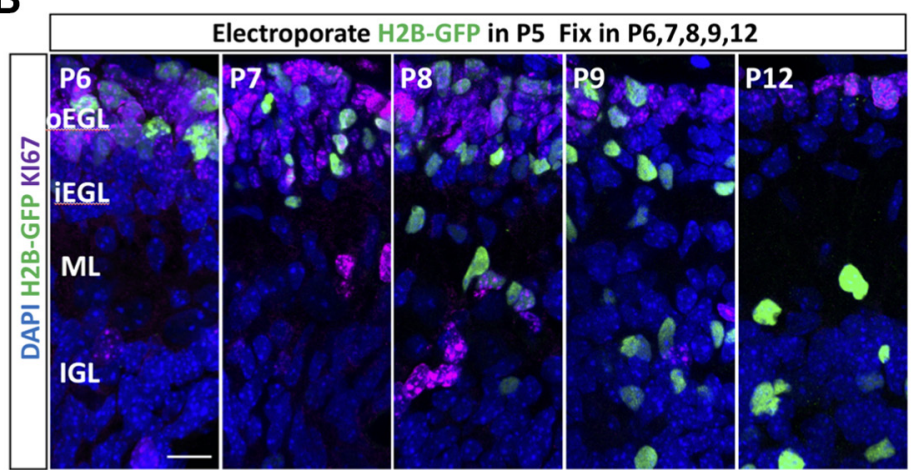

C

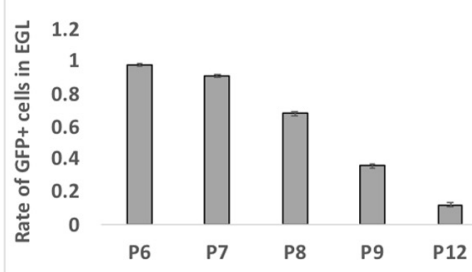

D

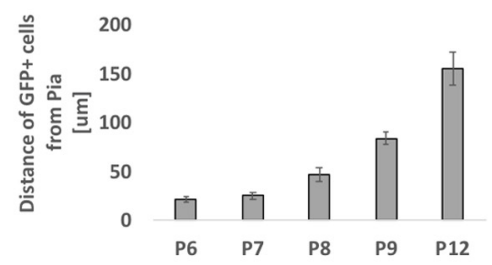

E

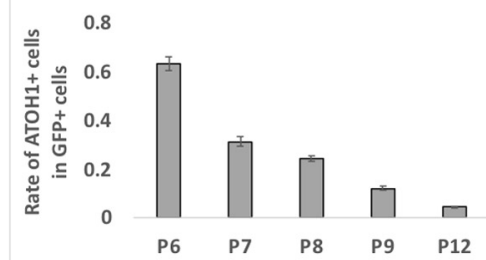

$\mathbf{F}$

Figure 5. Dynamics of electroporated cells in cerebellar development. $\boldsymbol{A}-\boldsymbol{F}, \mathrm{pCAG}-\mathrm{H} 2 \mathrm{~B}-\mathrm{GFP}$ was electroporated to P5 mouse EGL. Electroporated mice were fixed at P6, P7, P8, P9, or P12 and subjected to further analyses $(\boldsymbol{A}, \boldsymbol{B})$. Since GC-lineage cells migrate from EGL to IGL during their maturation (Schilling, 2018), we examined the rate of transfected cells in the EGL and distance of the transfected cell position from the pial matter $(\boldsymbol{C}, \boldsymbol{D})$. We also checked the rates of ATOH1-positive $(\boldsymbol{E})$ and KI67-positive $(\boldsymbol{F})$ cells in GFP-positive cells. Because plasmids were mainly introduced to Lobule IV/V or VI by electroporation in our experimental condition, all analyses in this study were conducted in Lobule IV/V or VI. Scale bars: $80 \mu \mathrm{m}(\boldsymbol{A})$ and $15 \mu \mathrm{m}(\boldsymbol{B})$.

the expression vector and then examined as they migrated inwardly as development proceeds (Fig. 5A-D). The rate of ATOH1 or KI67-positive cells in electroporated cells were gradually decreased (Fig. $5 E, F$ ), reflecting gradual differentiation from GCPs to GCs.

By this method, we introduced $K D$ vectors for Notch1 and Notch2 plus pCAG-H2B-GFP into the P6 cerebella and analyzed them at P9 by immunostaining with KI67 and ATOH1, markers for GCPs (Fig. 6A-C). Interestingly, KD for Notch2 significantly reduced the rates of ATOH1-positive cells as well as KI67-positive cells, while KD for Notch1 did not show significant effects (Fig. 6B,C). This suggests that Notch2 but not Notch1 is involved in suppressing differentiation of proliferative GCPs to postmitotic GCs putatively in a cell autonomous manner. Next, we electroporated an overexpression vector (Fig. 6D-F) or KD vectors (Fig. $6 G-K)$ for Hes1 plus pCAG-H2B-GFP into the P6 cerebella, which were fixed at $\mathrm{P} 8$ and $\mathrm{P} 9$, respectively. While overexpression of HES1 increased ATOH1-positive and KI67-positive cells, KD of Hes1 decreased those cells, implicating that HES1 suppresses differentiation from GCPs to GCs presumably in a cell autonomous manner. On the other hand, introduction of $\mathrm{KD}$ vectors for Hes5 did not affect the ATOH1 and KI67 positivity of GCPs (Fig. 7D-H). This suggests that HES5 may not be involved in suppression of GCP differentiation into GCs, consistent with our finding that Hes5 expression was very low compared with Hes 1 in GCP-like cells (Fig. 1C). Interestingly, introduction of a HES5 overexpression vector succeeded in suppressing differentiation of GCPs (Fig. $7 A-C$ ), similar to the effect of HES1. This led us to believe that, if overexpressed, HES5 has the ability to suppress GCP differentiation, probably via the same downstream pathway as that of HES1. However, as the expression of HES5 
A

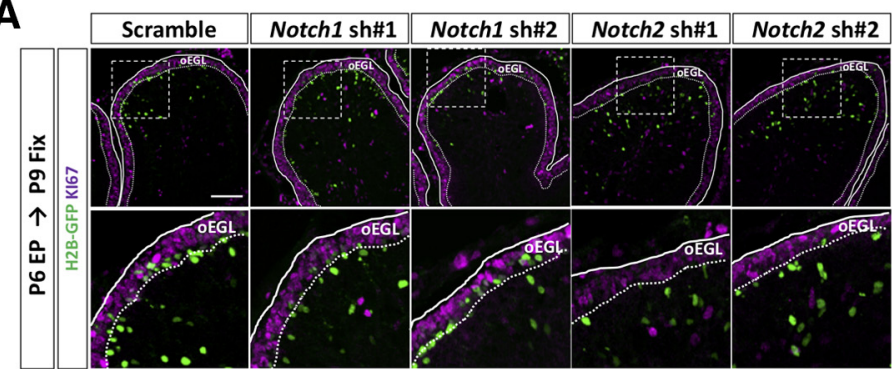

B

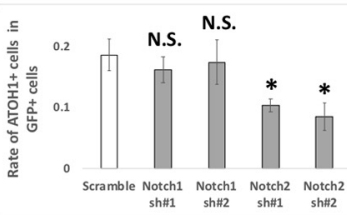

C

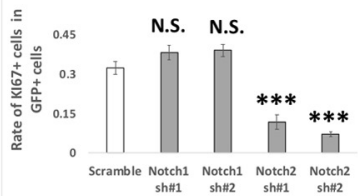

D

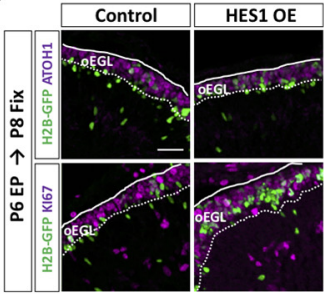

E

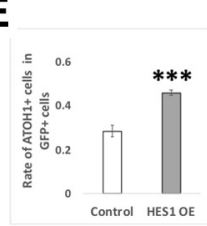

$\mathbf{F}$

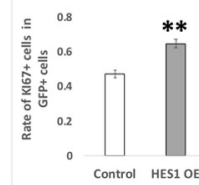

G

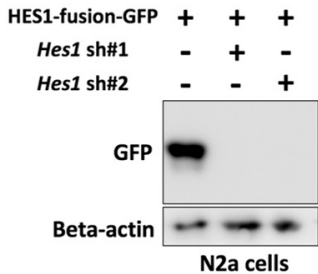

H

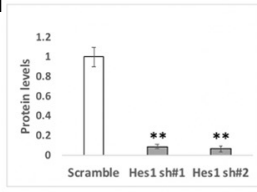

I

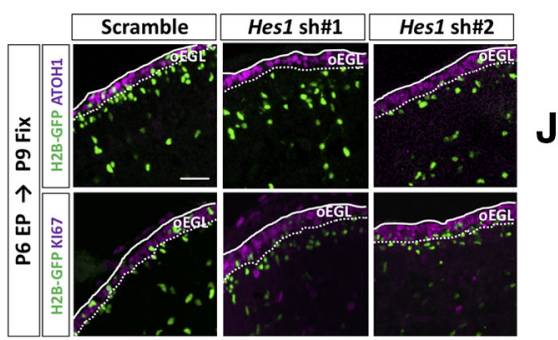

$\mathbf{J}$

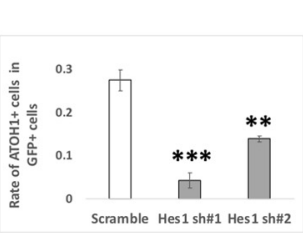

K

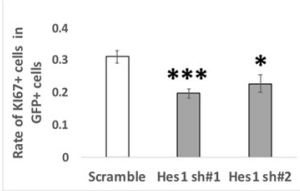

Figure 6. NOTCH2 and HES1 tend to maintain GCPs in immature and proliferative state. $\boldsymbol{A}-\boldsymbol{C}$, Electroporation of Notch1,2 kDa vectors plus pCAG-H2B-GFP into the EGL $(\boldsymbol{A})$. Differentiation of electroporated cells were evaluated with the expression of ATOH1 and KI67 (B, C). Animal numbers: $N=4$ for pU6-Notch1,2-sh\#1, $N=5$ for pU6-Notch1,2-sh\#2. Scale bars: 80 and $30 \mu \mathrm{m}(\boldsymbol{A})$. $\boldsymbol{D}-\boldsymbol{K}$, Electroporation with HES1 overexpression (OE) and KD vectors plus pCAG-H2B-GFP to the EGL. The rate of differentiation in electroporated cells was analyzed by immunohistochemistry with ATOH1 and $\mathrm{KI} 67(\boldsymbol{E}, \boldsymbol{F}, \boldsymbol{J}, \boldsymbol{K})$. The pCAG-empty vector $(\boldsymbol{D}-\boldsymbol{F})$ and the scrambled shRNA vector (Scramble) were used as controls (I-K). KD vectors for Hes1 were checked the efficiency in vitro (G, $\boldsymbol{H}$ ). CAG-HES1-fusion-GFP were co-transfected with Hes1 KD vectors to Neuro2a cells and the protein level were checked by Western blotting with GFP antibody $(\mathbf{G}, \boldsymbol{H})$. $\beta$-Actin was used as a reference. Animal numbers: $N=5$ for $(\boldsymbol{D}-\boldsymbol{F}), N=4$ for $(\boldsymbol{I}-\boldsymbol{K})$. Sample numbers: $N=3$ for $(\boldsymbol{G}, \boldsymbol{H})$. Scale bars: $30 \mu \mathrm{m}(\boldsymbol{D}, \boldsymbol{I})$. Data are shown as mean $\pm \mathrm{SEM} ;{ }^{*} p<0.05,{ }^{\star \star} p<0.01$, ${ }^{\star \star \star} p<0.001$, Student's $t$ test.

in GCPs is very low, endogenous HES5 is unlikely to be involved in that process. Altogether, these findings suggest that Notch2-dependent and Hes1-dependent Notch signaling is involved in maintaining GCPs in a proliferative and immature state in the developing cerebellum.

\section{JAG1 cell non-autonomously upregulates Notch signaling in surrounding GCPs}

Among the ligands for Notch signaling, we found that Jag1 was strongly expressed in GCP-like cells compared with Jag2, and DIl1,3,4 (Fig. 1C) and in the EGL (Fig. 1G). 


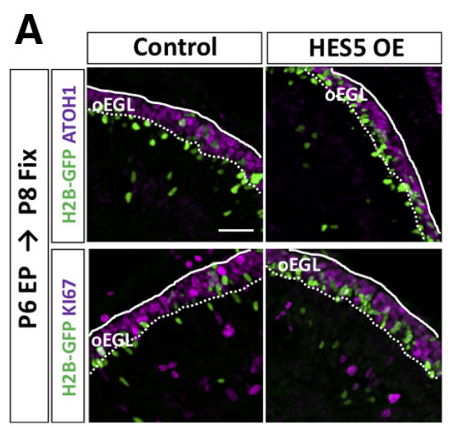

B

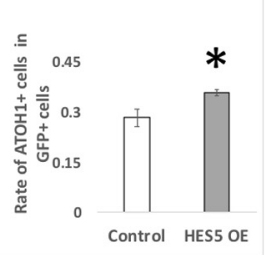

C

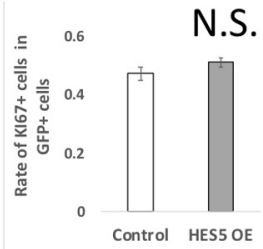

D

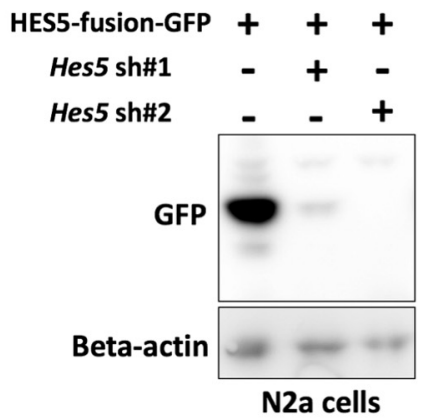

E

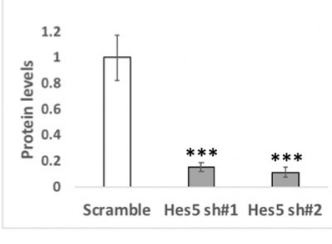

$\mathbf{F}$

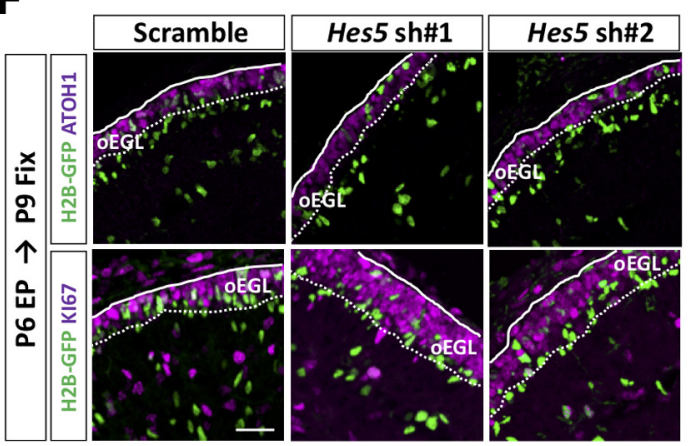

G

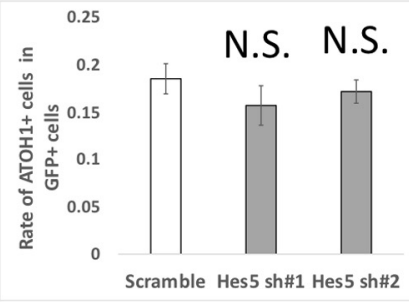

H

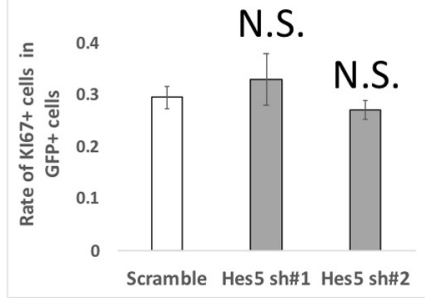

Figure 7. Endogenous HES5 is not physiologically involved in the differentiation of GCPs. $\boldsymbol{A}-\boldsymbol{H}$, Overexpression $(\boldsymbol{A}-\boldsymbol{C})$ and $\mathrm{KD}(\boldsymbol{F}-\boldsymbol{H})$ vectors for HES5 plus pCAG-H2B-GFP were electroporated to P6 cerebella, followed by fixation at indicated stages. Samples were immunostained with indicated antibodies and analyzed as in Figure $6 E, F, J, K$. The pCAG-empty vector $(\boldsymbol{A}-\boldsymbol{C})$ and Scramble $(\boldsymbol{F}-\boldsymbol{H})$ were used as controls. KD vectors for Hes5 were checked the efficiency in vitro $(\boldsymbol{D}, \boldsymbol{E})$. CAG-HES5-fusion-GFP were co-transfected with Hes5 KD vectors to Neuro2a cells and the protein level were checked by Western blotting with GFP antibody $(\boldsymbol{D}, \boldsymbol{E})$. $\beta$-Actin was used as a reference. Animal numbers: $N=5$ for pCAG-HES5 OE, $N=4$ for pU6-Hes5-sh. Sample numbers: $N=3$ for $(\boldsymbol{D}, \boldsymbol{E})$. Scale bars: $30 \mu \mathrm{m}(\boldsymbol{A}, \boldsymbol{F})$. Data are shown as mean \pm SEM; ${ }^{*} p<0.05$, Student's $t$ test.

Therefore, we performed overexpression and KD experiments for Jag1 with the same experimental strategy used for Notch and Hes genes (Fig. 6). While overexpression of JAG1 decreased rates of ATOH1 and KI67 positivity in the transfected cells (Fig. 8A-C), KD of Jag1 led to the opposite results (Fig. $8 D-H$ ). This suggests that Jag1 accelerates differentiation of Jag1-expressing GCPs into GCs, thus exhibiting contrasting effects to those of Notch2 and Hes1.

It is known that, in many tissues, JAG1 acts as a ligand for Notch receptor proteins to increase Notch signaling in surrounding cells (Gomi et al., 2016). In addition, we showed that Notch signaling activity can be monitored by pHes1-d2GFP in GCPs (Figs. 2A,G, 3A,C). Therefore, to test whether JAG1 increases Notch-signaling activity in surrounding cells, we performed a "double electroporation" experiment. We first electroporated JAG1 overexpression or control vector plus pCAG-H2B-BFP (nuclear localizing BFP) to $\mathrm{P} 6$ cerebella and performed the second electroporation with pHes1-d2GFP plus pCAG-mCherry to the same cerebella at $6 \mathrm{~h}$ after the first electroporation. The animals were killed, and cerebella were fixed at P8. Under this experimental condition, some cells were singly transfected by the first or the second electroporation and some were doubly labeled by the both electroporations (Fig. 9A). Among cells transfected by the second electroporation (mCherry+ cells), the rates of doubly electroporated cells (BFP-positive cells) were not significantly 


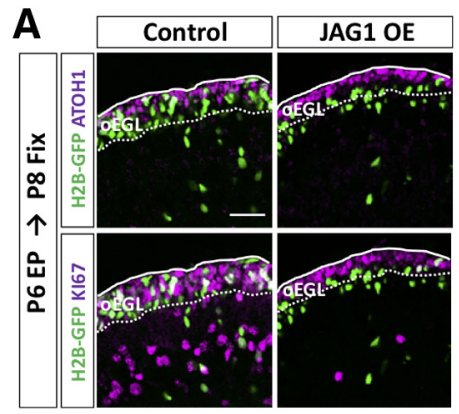

B

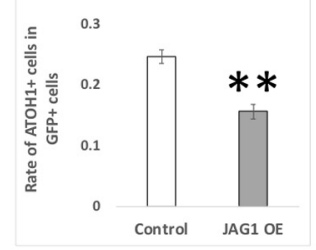

C

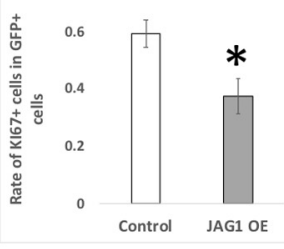

D

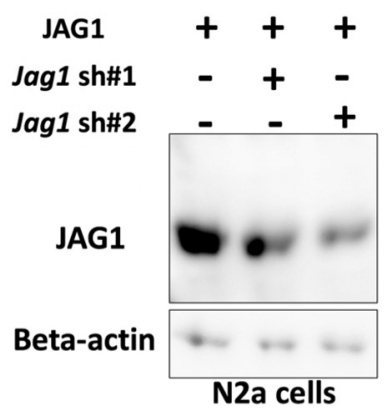

E
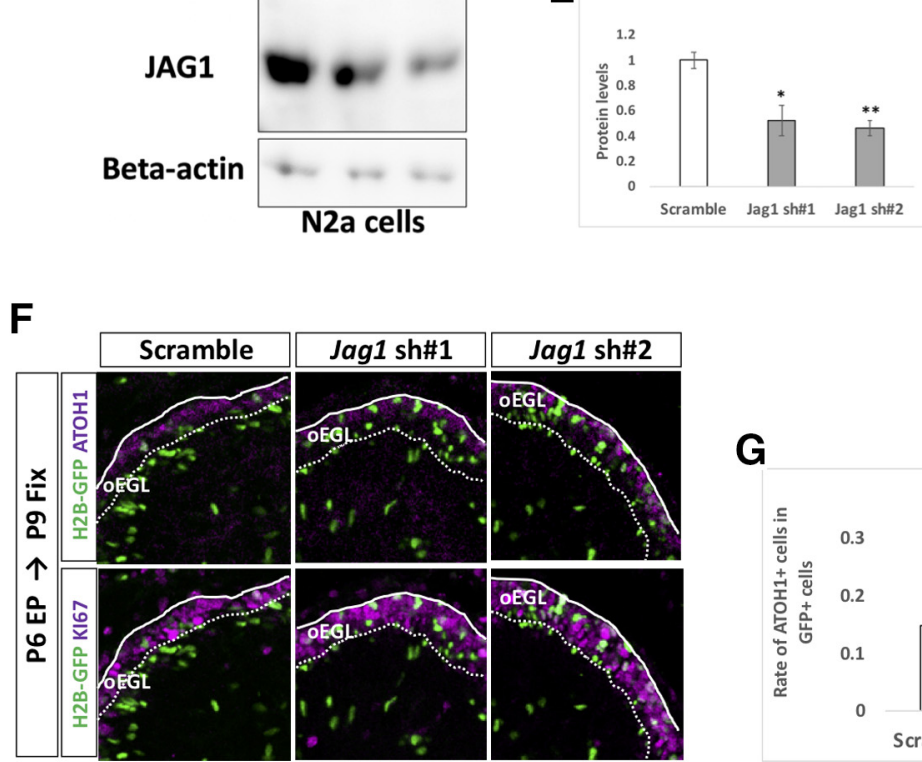

G

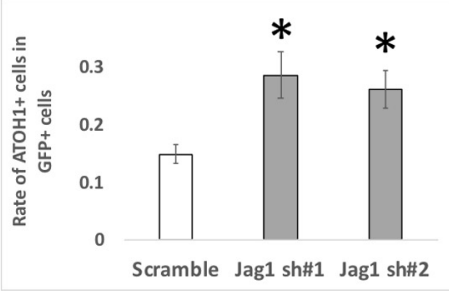

H

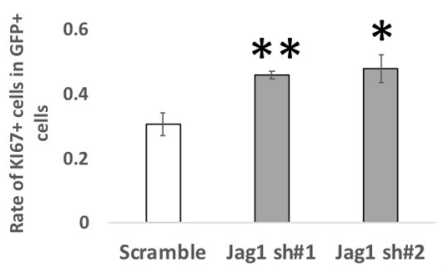

Figure 8. JAG1 cell autonomously promotes differentiation of GCPs. $\boldsymbol{A}-\boldsymbol{H}$, Electroporation with overexpression $(\boldsymbol{A}-\boldsymbol{C})$ and $\mathrm{KD}(\boldsymbol{F}-\boldsymbol{H})$ vectors for JAG1 plus pCAG-H2B-GFP into the P6 EGL. Electroporated cells were estimated by immunostaining for ATOH1 and $\mathrm{KI} 67$ at indicated developmental stages. The pCAG-empty vector $(\boldsymbol{A}-\boldsymbol{C})$ and Scramble $(\boldsymbol{F}-\boldsymbol{H})$ were used as controls. KD vectors for Jag1 were checked the efficiency in vitro $(\boldsymbol{D}, \boldsymbol{E})$. CAG-JAG1 were co-transfected with Jag1 KD vectors to Neuro2a cells and the protein level were checked by Western blotting with JAG1 antibody $(\boldsymbol{D}, \boldsymbol{E}) . \beta$-Actin was used as a reference. Animal numbers: $N=4$ for pCAG-JAG1 OE, pU6-Jag1-sh\#1, $n=5$ for pU6-Jag1-sh\#2. Sample numbers: $N=3$ for $(\boldsymbol{D}, \boldsymbol{E})$. Scale bars: $30 \mu \mathrm{m}(\boldsymbol{A}, \boldsymbol{F})$. Data are shown as mean \pm SEM; ${ }^{*} p<0.05,{ }^{* *} p<0.01$, Student's $t$ test.

different between the control and JAG1 introduced samples (Fig. 9C). We quantified GFP-positive cells in mCherry + cells that had been transfected in the second electroporation but not in the first electroporation (BFPnegative). Interestingly, GFP-positivity among those cells was increased when JAG1 was introduced at the first electroporation (Fig. 9A,B). This suggests that the JAG1 expression in GCPs increases Notch-signaling activity of surrounding GCPs.

Next, to examine the cell non-autonomous effect by KD for Jag1, we designed an experiment that combined electroporation and EdU-incorporation techniques. We electroporated the KD vector for Jag1 plus pCAG-H2B-GFP in GCPs of P5 mice with a simultaneous intraperitoneal injection of EdU and then fixed the samples at P8 for immunostaining (Fig. 9D). In this experiment, EdU-positive/ GFP-negative cells could be regarded as progeny cells of proliferative GCPs that had not been introduced with the Jag1 KD vector at P5. Furthermore, because GFP-positive cells were observed very densely in the electroporated EGL area (Fig. 9D), most or many of EdU-positive/ GFP-negative cells in the electroporated area are likely offspring cells of non-transfected GCPs that had a contact with Jag1 KD GCPs. As observed in Figure $8 F-H$, KI67-positive cells were also increased in Jag1 KD cells (GFP-positive cells) in this experiment (Fig. 9D,E). In 
A
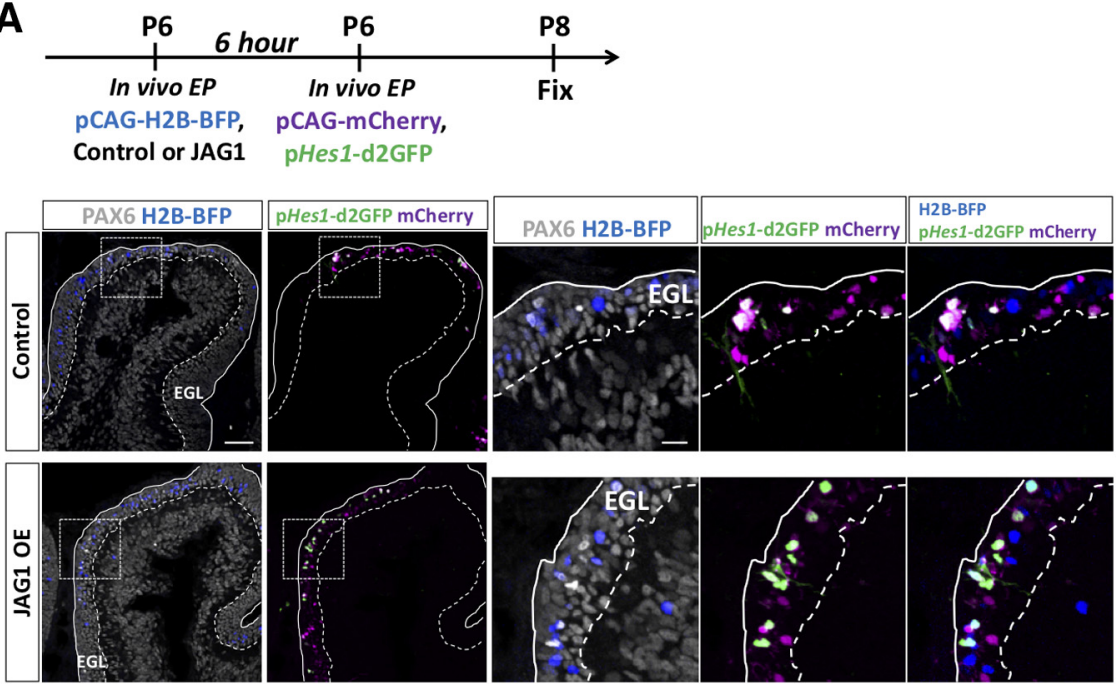

D
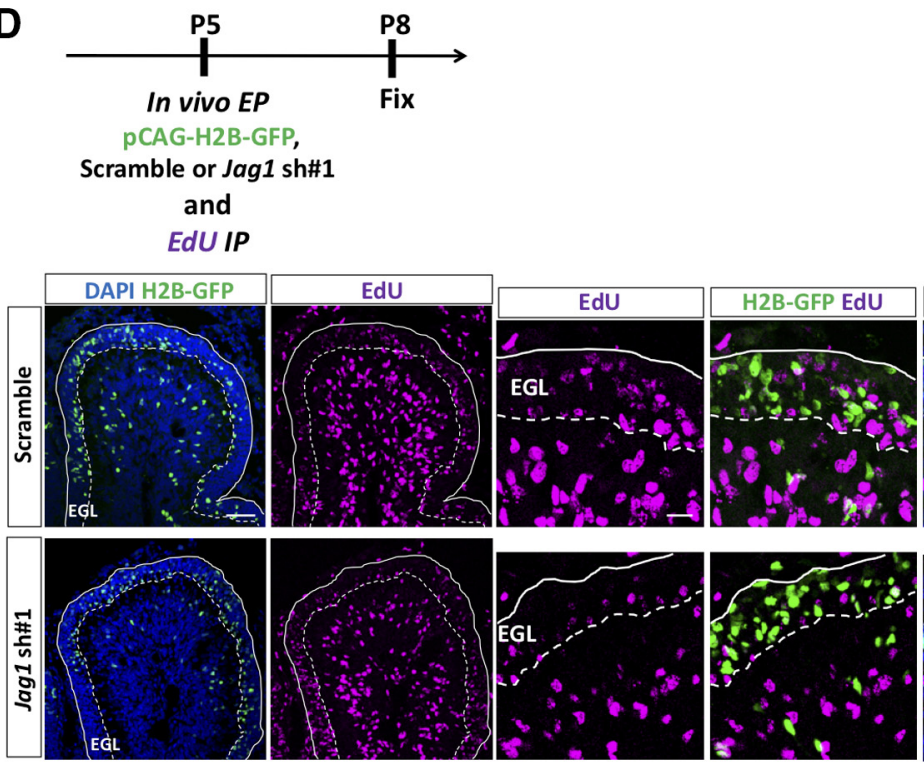

Figure 9. JAG1 cell non-autonomously upregulates Notch-signaling and suppresses differentiation of surrounding cells. $\boldsymbol{A}-\boldsymbol{C}$, Double electroporations with a 6-h interval were performed to P6 EGL according to the indicated schedule. Rate of GFP+ cells in mCherry+ cells was drastically increased in JAG1-overexpressed (OE) mice compared with control (A, B). Rates (20-30\%) of the co-electroporated cells (BFP+ cells in mCherry+ cells) were not significantly different in control and JAG1 OE (A, C). BFP-double positive and mCherry-double positive co-electroporated cells were excluded in the analysis of Figure $5 B$. Animal numbers: $N=4$. Scale bars: 80 and $30 \mu \mathrm{m}(\boldsymbol{A})$. $\boldsymbol{D}-\boldsymbol{F}$, Electroporation with indicated vectors and intraperitoneal administration of EdU were performed in P5 mice, followed by fixation at P8. GFP+ cells were Jag1-downregulated cells and EdU+ GFP- cells were presumed neighboring cells. In the Jag1 KD mice, the rate of GCPs in the signal sending cells were increased ( $\boldsymbol{D}, \boldsymbol{E})$. In contrast, differentiation of GCPs was accelerated in Jag1 KD mice compared with control mice $(\boldsymbol{D}, \boldsymbol{F})$. Animal numbers: $N=4$. Scale bars: 80 and $30 \mu \mathrm{m}(\boldsymbol{D})$. Data are shown as mean $\pm \mathrm{SEM} ;{ }^{\star \star} p<0.01,{ }^{\star \star \star} p<0.001$, Student's $t$ test.

contrast, the rate of KI67-positive cells was decreased in EdU-positive/GFP-negative cells (Fig. 9D,F). This implies that Jag1 KD promotes differentiation of surrounding cells. Furthermore, together with the findings that JAG1 overexpression enhances Notch activity of surrounding cells (Fig. 9A-C) and that Notch2-Hes1-dependent Notch signaling keeps GCPs in an immature and proliferative state, this suggests that JAG1-expressing GCPs may maintain surrounding GCPs in a proliferative state via NOTCH2-HES1 pathway-dependent Notch signaling (Fig. 10K).

\section{Hes 1 is involved in downregulating NEUROD1 expression}

It has been suggested that the transcription factor, NEUROD1, is required for differentiation of GCs in the developing cerebellum (Pan et al., 2009). Consistent with this, in our experimental conditions, overexpression of 


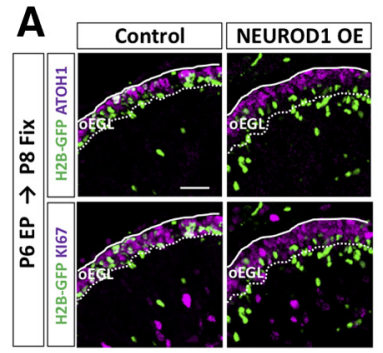

B

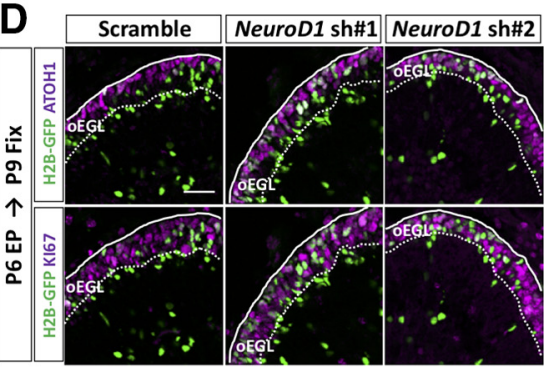

G

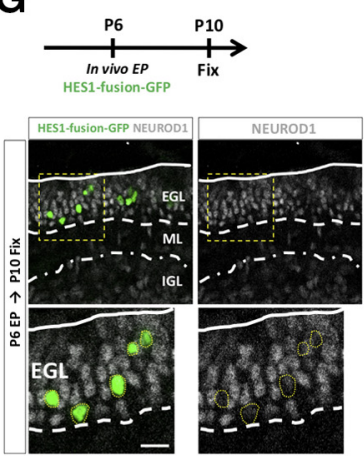

E

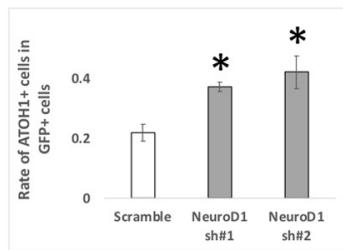

$\mathbf{F}$

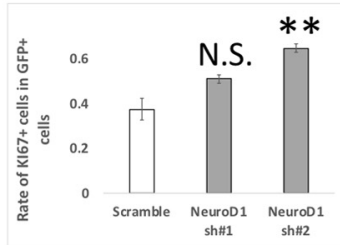

\section{H}

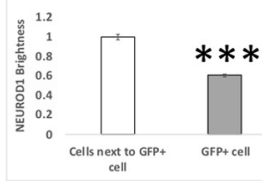

C

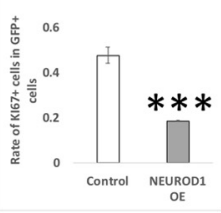

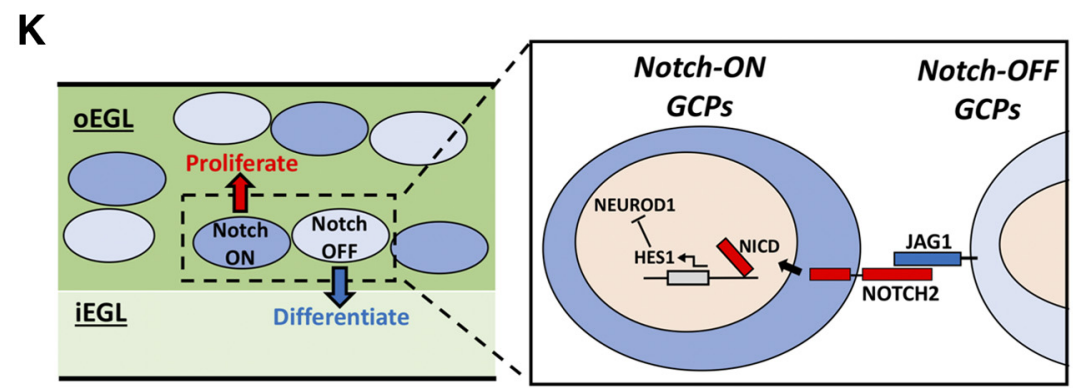

Figure 10. HES1 downregulates NEUROD1 in GCPs/GCs lineage. $\boldsymbol{A}-\boldsymbol{F}$, Overexpression and KD vectors plus pCAG-H2B-GFP were electroporated to P6 cerebella, followed by fixation at indicated stages. Samples were immunostained with indicated antibodies and analyzed as in Figure 3E, $F, H, I$. Animal numbers: $n=4$ for pCAG-NEUROD1 OE, pU6-NeuroD1-sh\#1,2. Scale bars: $30 \mu m(\boldsymbol{A}, \boldsymbol{D})$. Data are shown as mean \pm SEM; ${ }^{\star} p<0.05,{ }^{* *} p<0.01,{ }^{* \star *} p<0.001$, Student's $t$ test. $\boldsymbol{G}, \boldsymbol{H}$, HES1-fusion-GFP was electroporated into P6 EGL and cerebella fixed at P10 (G). The fluorescence intensities for NEUROD1 were estimated in transfected (GFP-positive) cells $(\boldsymbol{H})$. Animal numbers: $N=4$. Scale bars: 30 and $10 \mu \mathrm{m}(\boldsymbol{A}) . \boldsymbol{I}, \boldsymbol{J}$, Hes1 KD and pCAG-H2B-GFP were co-electroporated into P6 EGL, followed by immunostaining at P9 (I). Immunofluorescence intensities for NEUROD1 were estimated (I, $\boldsymbol{J})$. Animal numbers: $N=4$. Scale bars: 30 and $10 \mu \mathrm{m}(\boldsymbol{A})$. Data are shown as mean \pm SEM; ${ }^{* \star} p<0.001$, Student's $t$ test. $\boldsymbol{K}$, A schematic model for Notch signaling in GCPs. In the oEGL of the developing cerebellum, there are two types of GCPs, Notch-signaling ON (signal receiving) and OFF (signal sending) GCPs. JAG1 expressed on the signal-sending GCPs interacts with NOTCH2 on the signal-receiving GCPs. This interaction induces cleavage of NICD by a $\gamma$ secretase to upregulate Hes 1 expression in the signal-receiving GCPs. HES1 suppresses expression of NEUROD1, eventually maintaining GCPs at immature and proliferative status. Signal-sending GCPs express only a small amount of HES, resulting in expression of NEUROD1 and subsequent differentiation into GCs. 
NEUROD1 significantly decreased ATOH1-positive cells and KI67-positive cells (Fig. 10A-C). Introduction of KD vectors for NeuroD1 caused the opposite results to the overexpression experiment (Fig. 10D-F). These observations confirmed that NEUROD1 is involved in promoting the differentiation of GCPs to GCs. To investigate the relationship between Notch signaling and NEUROD1 expression, we electroporated pCAG-HES1-fusion-GFP (HES1-GFP fusion protein) into GCPs at P6 and fixed the cerebella at P10. HES1-GFP overexpression reduced the immunofluorescence signals for NEUROD1 in the EGL (Fig. 10G,H). In contrast, Hes1 KD displayed the opposite results (Fig. 10/,ل). These observations suggest that Hes1 maintains the proliferative and immature states of GCs via regulation of NEUROD1.

\section{Discussion}

During cerebellar development, numerous GCPs, packed in the oEGL, seem to constitute a uniform population. However, the molecular regulatory machinery underlying how maternal GCPs produce proportional numbers of sister GCPs and GCs has not been well understood. In this study, we found that there are two populations of GCPs in the oEGL, Notch-signaling ON and OFF cells. The former possess more immature and proliferative characteristics, while the latter have more differentiative and less proliferative features. Expression of JAG1 in GCPs affects surrounding cells to become Notch-signaling ON cells via the Notch2-Hes1 pathway. HES1 decreases NEUROD1 expression in the ON-GCPs that eventually differentiate into GCs.

Several studies have reported that Notch-related transcripts and proteins are expressed in the postnatal cerebellum by means of RT-PCR (Solecki et al., 2001), in situ hybridization (Tanaka et al., 1999; Irvin et al., 2001, 2004; Solecki et al., 2001; Stump et al., 2002; Eiraku et al., 2005), and immunostaining (Tanaka and Marunouchi, 2003). However, some of the data from these studies were contradictory. This might be caused by differences of experimental conditions and/or differences in the cell population purity. In this study, we aimed to distinctly investigate the gene expression in GCPs and GCs with the hypothesis that Notch-related molecules might be differentially expressed between the two cell types. We successfully purified GCP and GC-like cells (Kutscher et al., 2020). By quantitative RT-PCR analyses as well as immunohistochemistry, our results suggest that Notch1, Notch2, Hes1, and Jag1 were prominently expressed in GCPs, while Hes5, Jag2, and DIl1 were significantly expressed in GCs. This differential expression between GCPs and GCs seems to partly explain the previous contradictory findings.

Several studies have previously reported phenotypes of knock-out (KO) mice for Notch-related genes (Eiraku et al., 2005; Weller et al., 2006; Komine et al., 2007; Hiraoka et al., 2013) during postnatal cerebellar development. Conditional KO (cKO) of Notch1, Notch2, RBPJ, or DIl in astroglial cell lineage with a GFAP-Cre line resulted in disorganized positioning and morphology of Bergmann glia (BG; Komine et al., 2007; Hiraoka et al., 2013), although
GCP/GC-related phenotypes were not assessed in those reports. In cKO mice for Jag1 in the whole cerebellum, generated by crossing with an En2-Cre line, the position and morphology of BGs were also impaired. In those mice, the EGL was abnormally retained until P20, because of the aberrant GC migration (Weller et al., 2006). However, since Jag1 is expressed in both BGs and GCPs/GCs, it was unclear whether Jag1 expression in BGs or GCPs/GCs were responsible for the phenotype. In this study, by in vivo electroporation KD experiments, we clearly showed that Jag1 expression in GCPs is involved in GCP differentiation. Another group analyzed conventional KO mice for Dner, a non-canonical ligand for Notch signaling, expressed in Purkinje cells (PCs) as well as GCs in the iEGL (Eiraku et al., 2002, 2005). In the mutant cerebellum, localization and morphology of BGs are impaired, while GC migration was delayed. The authors suggest that Notch signaling via DNER on PCs and NOTCH1 on $B G$ sight be involved in $B G$ differentiation, although there still remains the possibility that DNER expressed in GCs are responsible for the phenotype. In another in vitro cell and explant culturing study, it was reported that overexpression of NOTCH2 and HES1 suppressed process extension of GCs (Solecki et al., 2001). Although this data showed the ability of these molecules to affect GC differentiation, their physiological requirement remained unknown because of the lack of loss of function experiments.

Despite these previous reports on Notch signaling molecules, Notch activity has not been detected in postnatal cerebellar development. By monitoring promoter activities, we found the presence of Hes1-dependent, but not Hes5-dependent, Notch signaling activity in GCPs of the oEGL. This is the first report to directly show the presence of Notch signaling in the cerebellar EGL. Around half of GCPs are Notch-ON GCPs, while the others are NotchOFF GCPs. In silico analyses suggested that the former were more immature and proliferative, while the latter possessed opposite features. KD and overexpression experiments by electroporation showed that NOTCH2 and HES1 are cell-autonomously required for Notch activity in GCPs. On the other hand, JAG1 cell non-autonomously upregulates the Notch activities of surrounding GCPs. Despite its expression in GCPs, NOTCH1 was not involved in the Hes1-dependent Notch signaling activity. It might be possible that NOTCH1 activates different downstream Hes-family genes, such as Hey.

In early neural development, Hes 1 is known to maintain stemness of mouse neural progenitor cells (NPCs) by suppressing the expression of proneural genes, such as Ascl1 (Achaete-scute family bHLH transcription factor 1) and Ngn2 (Neurogenin 2; Kageyama et al., 2007). In mouse ventral telencephalon NPCs, there is oscillating expression of bHLH transcriptional genes Hes1, Ascl1, and Olig2 (Imayoshi et al., 2013). Once the fluctuation is lost and the expression of the genes are sustained, cell fates are determined to be astrocytes, neurons or oligodendrocytes (Imayoshi et al., 2013). This oscillation system may enable creation of minor differences within a uniform cell population, and eventually generate plural cell fates. Although we do not have any direct evidence, it 
is possible that Notch signaling also fluctuates among cerebellar GCPs during development. However, even if this was the case, the transition from ON to OFF or OFF to ON might occur very quickly, as we can barely detect the transient state GCPs, or intermediate promoter activities for Hes 1 as visualized by pHes1-d2GFP (Fig. 3D,E). In addition, since Hes1 promoter activities were not detected in mature GCs in the IGL, Notch signaling is thought to be fixed in the OFF state after GC differentiation.

The SHH subgroup of medulloblastoma, one of the major pediatric brain tumors, is known to be derived from the GC lineage, that is, GCPs/GCs (Goodrich et al., 1997). The Eberhart group previously showed that expression of Notch2 but not Notch1 was upregulated in medulloblastoma compared with normal pediatric cerebella, although they did not discriminate between $\mathrm{SHH}$ and the other subgroups (Fan et al., 2004). They also showed that Notch2 and Hes 1 are involved in the proliferation of medulloblastoma-derived cell line. These observations, at least in part, seem to be consistent with our finding that the Jag1Notch2-Hes1 pathway maintains GCPs in a proliferative state by upregulating Notch signaling. Therefore, this study may provide clues to understanding the mechanisms underlying tumorigenesis or growth of medulloblastoma and to finding potential therapeutic vulnerabilities.

In this study, we first visualized Notch-signaling in GCPs. NOTCH2 and HES1 are involved in Notch-signaling to maintain GCPs in an immature and proliferative state in a cell autonomous manner. This system may generate two distinct types of GCPs, NOTCH-ON and OFF, and contribute to production of an appropriate balance of sister GCPs and GCs from mother GCPs, eventually leading to the formation of the normal cerebellum. We believe that this study gives insights into understanding the basic machinery to produce different cell types from a seemingly uniform cell population in normal cerebellar development and also the pathology of medulloblastoma.

\section{References}

Anders S, Pyl PT, Huber W (2015) HTSeq-a Python framework to work with high-throughput sequencing data. Bioinformatics 31:166-169.

Andersson ER, Sandberg R, Lendahl U (2011) Notch signaling: simplicity in design, versatility in function. Development 138:35933612.

Basson MA, Wingate RJ (2013) Congenital hypoplasia of the cerebellum: developmental causes and behavioral consequences. Front Neuroanat 7:29.

Campos-Ortega J, Hartenstein AV (1985) The embryonic development of Drosophila melanogaster. Berlin; Heidelberg: Springer.

Chang $\mathrm{CH}$, Zanini M, Shirvani $\mathrm{H}$, Cheng JS, Yu H, Feng $\mathrm{CH}$, Mercier AL, Hung SY, Forget A, Wang CH, Cigna SM, Lu IL, Chen WY, Leboucher S, Wang WJ, Ruat M, Spassky N, Tsai JW, Ayrault O (2019) Atoh1 controls primary cilia formation to allow for SHH-triggered granule neuron progenitor proliferation. Dev Cell 48:184199.e5.

Chizhikov V, Millen KJ (2003) Development and malformations of the cerebellum in mice. Mol Genet Metab 80:54-65.

Dobin A, Davis CA, Schlesinger F, Drenkow J, Zaleski C, Jha S, Batut P, Chaisson M, Gingeras TR (2013) STAR: ultrafast universal RNA-seq aligner. Bioinformatics 29:15-21.

Eiraku M, Hirata Y, Takeshima H, Hirano T, Kengaku M (2002) Delta/ Notch-like epidermal growth factor (EGF)-related receptor, a novel
EGF-like repeat-containing protein targeted to dendrites of developing and adult central nervous system neurons. J Biol Chem 277:25400-25407.

Eiraku M, Tohgo A, Ono K, Kaneko M, Fujishima K, Hirano T, Kengaku M (2005) DNER acts as a neuron-specific Notch ligand during Bergmann glial development. Nat Neurosci 8:873-880.

Fan X, Mikolaenko I, Elhassan I, Ni XZ, Wang Y, Ball D, Brat DJ, Perry A, Eberhart CG (2004) Notch1 and Notch2 have opposite effects on embryonal brain tumor growth. Cancer Res 64:7787-7793.

Gomi K, Staudt MR, Salit J, Kaner RJ, Heldrich J, Rogalski AM, Arbelaez V, Crystal RG, Walters MS (2016) JAG1-mediated Notch signaling regulates secretory cell differentiation of the human airway epithelium. Stem Cell Rev Rep 12:454-463.

Goodrich LV, Milenković L, Higgins KM, Scott MP (1997) Altered neural cell fates and medulloblastoma in mouse patched mutants. Science 277:1109-1113.

Hiraoka Y, Komine O, Nagaoka M, Bai N, Hozumi K, Tanaka K (2013) Delta-like 1 regulates Bergmann glial monolayer formation during cerebellar development. Mol Brain 6:25.

Imayoshi I, Isomura A, Harima Y, Kawaguchi K, Kori H, Miyachi H, Fujiwara T, Ishidate F, Kageyama R (2013) Oscillatory control of factors determining multipotency and fate in mouse neural progenitors. Science 342:1203-1208.

Irvin DK, Zurcher SD, Nguyen T, Weinmaster G, Kornblum HI (2001) Expression patterns of Notch1, Notch2, and Notch3 suggest multiple functional roles for the Notch-DSL signaling system during brain development. J Comp Neurol 436:167-181.

Irvin DK, Nakano I, Paucar A, Kornblum HI (2004) Patterns of Jagged1, Jagged2, delta-like 1 and delta-like 3 expression during late embryonic and postnatal brain development suggest multiple functional roles in progenitors and differentiated cells. J Neurosci Res 75:330-343.

Kageyama R, Ohtsuka T, Kobayashi T (2007) The Hes gene family: repressors and oscillators that orchestrate embryogenesis. Development 134:1243-1251.

Kawauchi T, Chihama K, Nabeshima Y, Hoshino M (2006) Cdk5 phosphorylates and stabilizes p27kip1 contributing to actin organization and cortical neuronal migration. Nat Cell Biol 8:17-26.

Kawauchi D, Robinson G, Uziel T, Gibson P, Rehg J, Gao C, Finkelstein D, Qu C, Pounds S, Ellison DW, Gilbertson RJ, Roussel MF (2012) A mouse model of the most aggressive subgroup of human medulloblastoma. Cancer Cell 21:168-180.

Kohyama J, Tokunaga A, Fujita Y, Miyoshi H, Nagai T, Miyawaki A, Nakao K, Matsuzaki Y, Okano H (2005) Visualization of spatiotemporal activation of Notch signaling: live monitoring and significance in neural development. Dev Biol 286:311-325.

Komine O, Nagaoka M, Watase K, Gutmann DH, Tanigaki K, Honjo T, Radtke F, Saito T, Chiba S, Tanaka K (2007) The monolayer formation of Bergmann glial cells is regulated by Notch/RBP-J signaling. Dev Biol 311:238-250.

Kutscher LM, Okonechnikov K, Batora NV, Clark J, Silva PBG, Vouri M, van Rijn S, Sieber L, Statz B, Gearhart MD, Shiraishi R, Mack N, Orr BA, Korshunov A, Gudenas BL, Smith KS, Mercier AL, Ayrault O, Hoshino M, Kool M, et al. (2020) Functional loss of a non-canonical BCOR-PRC1.1 complex accelerates SHH-driven medulloblastoma formation. Genes Dev 34:1161-1176.

Lackey EP, Heck DH, Sillitoe RV (2018) Recent advances in understanding the mechanisms of cerebellar granule cell development and function and their contribution to behavior. F1000Res 7:1142.

Lehmann R, Jiménez F, Dietrich U, Campos-Ortega JA (1983) On the phenotype and development of mutants of early neurogenesis in Drosophila melanogaster. Wilehm Roux Arch Dev Biol 192:62-74.

Louvi A, Tsakonas SA (2006) Notch signaling in vertebrate neural development. Nat Rev Neurosci 7:93-102.

Love MI, Anders S, Huber W (2014) Differential analysis of count data - the DESeq2 package. Genome Biol 15:550.

Luistro L, He W, Smith M, Packman K, Vilenchik M, Carvajal D, Roberts J, Cai J, B-Fessler W, Hilton H, Linn M, Flohr A, Røtne RJ, Jacobsen H, Glenn K, Heimbrook D, Boylan JF (2009) Preclinical profile of a potent $\gamma$-secretase inhibitor targeting Notch signaling 
with in vivo efficacy and pharmacodynamic properties. Cancer Res 69:7672-7680.

Miyashita S, Adachi T, Yamashita M, Sota T, Hoshino M (2017) Dynamics of the cell division orientation of granule cell precursors during cerebellar development. Mech Dev 147:1-7.

Miyashita S, Owa T, Seto Y, Yamashita M, Aida S, Nishioka T, Kaibuchi K, Kawaguchi Y, Taya S, Hoshino M (2020) CyclinD1 controls development of cerebellar granule cell progenitors through phosphorylation and stabilization of ATOH1. EMBO J, in press.

Naito Y, Yoshimura J, Morishita S, Ui-Tei K (2009) siDirect 2.0: updated software for designing functional siRNA with reduced seeddependent off-target effect. BMC Bioinformatics 10:392.

Ohtsuka T, Imayoshi I, Shimojo H, Nishi E, Kageyama R, McConnell SK (2006) Visualization of embryonic neural stem cells using Hes promoters in transgenic mice. Mol Cell Neurosci 31:109-122.

Okonechnikov K, Conesa A, Alcalde FG (2016) Qualimap 2: advanced multi-sample quality control for high-throughput sequencing data. Bioinformatics 32:292-294.

Owa T, Taya S, Miyashita S, Yamashita M, Adachi T, Yamada K, Yokoyama M, Aida S, Nishioka T, Inoue YU, Goitsuka R, Nakamura T, Inoue T, Kaibuchi K, Hoshino M (2018) Meis1 coordinates cerebellar granule cell development by regulating Pax6 transcription, BMP signaling and Atoh1 degradation. J Neurosci 38:1277-1294.

Pan N, Jahan I, Lee JE, Fritzsch B (2009) Defects in the cerebella of conditional Neurod1 null mice correlate with effective Tg(Atoh1cre) recombination and granule cell requirements for Neurod1 for differentiation. Cell Tissue Res 337:407-428.

Schilling K (2018) Moving into shape: cell migration during the development and histogenesis of the cerebellum. Histochem Cell Biol 150:13-36.

Seto Y, Nakatani T, Masuyama N, Taya S, Kumai M, Minaki Y, Hamaguchi A, Inoue $\mathrm{YU}$, Inoue T, Miyashita S, Fujiyama T, Yamada M, Chapman H, Campbell K, Magnuson MA, Wright CV, Kawaguchi Y, Ikenaka K, Takebayashi H, Ishiwata S, et al. (2014) Temporal identity transition from Purkinje cell progenitors to GABAergic interneuron progenitors in the cerebellum. Nat Commun 5:3337.

Shitamukai A, Konno D, Matsuzaki F (2011) Oblique radial glial divisions in the developing mouse neocortex induce self-renewing progenitors outside the germinal zone that resemble primate outer subventricular zone progenitors. J Neurosci 31:3683-3695.
Shiraishi RD, Miyashita S, Yamashita M, Adachi T, Shimoda MM, Owa T, Hoshino M (2019) Expression of transcription factors and signaling molecules in the cerebellar granule cell development. Gene Expr Patterns 34:119068.

Solecki DJ, Liu XL, Tomoda T, Fang Y, Hatten ME (2001) Activated Notch2 signaling inhibits differentiation of cerebellar granule neuron precursors by maintaining proliferation. Neuron 31:557-568.

Stuart T, Butler A, Hoffman P, Hafemeister C, Papalexi E, Mauck WM, Hao Y, Stoeckius M, Smibert P, Satija R (2019) Comprehensive integration of single-cell data. Cell 177:18881902.e21.

Stump G, Durrer A, Klein AK, Lütolf S, Suter U, Taylor V (2002) Notch1 and its ligands Delta-like and Jagged are expressed and active in distinct cell populations in the postnatal mouse brain. Mech Dev 114:153-159.

Tanaka M, Marunouchi T (2003) Immunohistochemical localization of Notch receptors and their ligands in the postnatally developing rat cerebellum. Neurosci Lett 353:87-90.

Tanaka M, Kadokawa Y, Hamada Y, Marunouchi T (1999) Notch2 expression negatively correlates with glia differentiation in the postnatal mouse brain. J Neurobiol 41:524-539.

Thomas KC, Zheng XF, Suarez FG, Raftery JM, Quinlan KGR, Yang N, North KN, Houweling PJ (2014) Evidence based selection of commonly used RT-qPCR reference genes for the analysis of mouse skeletal muscle. PLoS One 9:e88653.

Tsakonas SA, Rand MD, Lake RJ (1999) Notch signaling: cell fate control and signal integration in development. Science 284:770776.

Weller M, Krautler N, Mantei N, Suter U, Taylor V (2006) Jagged1 ablation results in cerebellar granule cell migration defects and depletion of Bergmann glia. Dev Neurosci 28:70-80.

Williams RW, Herrup K (1988) The control of neuron number. Annu Rev Neurosci 11:423-453.

Yamada M, Seto Y, Taya S, Owa T, Inoue YU, Inoue T, Kawaguchi Y, Nabeshima Y, Hoshino M (2014) Specification of spatial identities of cerebellar neuron progenitors by ptf1a and atoh1 for proper production of GABAergic and glutamatergic neurons. J Neurosci 34:4786-4800.

Yang R, Wang M, Huang J, Yang XR, Gao WQ (2015) Cell division mode change mediates the regulation of cerebellar granule neurogenesis controlled by the sonic hedgehog signaling. Stem Cell Reports 5:816-828. 